\title{
Acetic Acid-Producing Endophyte Lysinibacillus fusiformis Orchestrates Jasmonic Acid Signaling and Contributes to Repression of Cadmium Uptake in Tomato Plants
}

\section{OPEN ACCESS}

Edited by:

Katharina Pawlowski,

Stockholm University, Sweden

Reviewed by:

Kinga Dziurka,

The Franciszek Górski Institute of Plant Physiology (PAS), Poland Ana Cristina Esteves,

University of Aveiro, Portugal Mona Hassan Soliman, Cairo University, Egypt

*Correspondence:

Songhua Wang wangsh@ahstu.edu.cn Cheng Zhou

zhoucheng@njau.edu.cn

Specialty section: This article was submitted to Plant Symbiotic Interactions, a section of the journal Frontiers in Plant Science

Received: 20 February 2021 Accepted: 10 May 2021 Published: 04 June 2021

Citation:

Zhu L, Guo J, Sun Y, Wang S and Zhou C (2021) Acetic Acid-Producing

Endophyte Lysinibacillus fusiformis Orchestrates Jasmonic Acid Signaling and Contributes to Repression of Cadmium Uptake in Tomato Plants.

Front. Plant Sci. 12:670216. doi: 10.3389/fpls.2021.670216

\begin{abstract}
Lin Zhu',2, Jiansheng Guo ${ }^{2,3}$, Yujun Sun ${ }^{1}$, Songhua Wang ${ }^{1 *}$ and Cheng Zhou ${ }^{4 *}$
${ }^{1}$ Key Lab of Bio-Organic Fertilizer Creation, Ministry of Agriculture and Rural Affairs, Anhui Science and Technology University, Bengbu, China, ${ }^{2}$ School of Life Sciences and Technology, Tongji University, Shanghai, China, ${ }^{3}$ School of Medicine, Zhejiang University, Hangzhou, China, ${ }^{4}$ Jiangsu Provincial Key Lab of Solid Organic Waste Utilization, Jiangsu Collaborative Innovation Center of Solid Organic Wastes, Educational Ministry Engineering Center of Resource-Saving Fertilizers, Nanjing Agricultural University, Nanjing, China
\end{abstract}

Diverse signaling pathways regulated by phytohormones are essential for the adaptation of plants to adverse environments. Root endophytic bacteria can manipulate hormone-related pathways to benefit their host plants under stress conditions, but the mechanisms underlying endophyte-mediated plant stress adaptation remain poorly discerned. Herein, the acetic acid-producing endophytic bacteria Lysinibacillus fusiformis Cr33 greatly reduced cadmium (Cd) accumulation in tomato plants. L. fusiformis led to a marked increase in jasmonic acid (JA) content and downregulation of iron (Fe) uptake-related genes in Cd-exposed roots. Accordantly, acetic acid treatment considerably increased the JA content and inhibited root uptake of $\mathrm{Cd}$ uptake. In addition, the Cr33-inoculated roots displayed the increased availability of cell wall and rhizospheric Fe. Inoculation with Cr33 notably reduced the production of nitric oxide (NO) and suppressed Fe uptake systems in the Cd-treated roots, thereby contributing to hampering $\mathrm{Cd}$ absorption. Similar results were also observed for $\mathrm{Cd}$ treated tomato plants in the presence of exogenous JA or acetic acid. However, chemical inhibition of JA biosynthesis greatly weakened the endophyte-alleviated $\mathrm{Cd}$ toxicity in the plants. Collectively, our findings indicated that the endophytic bacteria L. fusiformis effectively prevented $\mathrm{Cd}$ uptake in plants via the activation of acetic acid-mediated JA signaling pathways.

Keywords: endophytes, jasmonic acid, cadmium toxicity, iron deficiency, acetic acid-producing bacteria

\section{INTRODUCTION}

Massive industrial waste and the use of phosphate fertilizers cause heavy metal pollution in agricultural soils (Sarwar et al., 2017). Soil cadmium (Cd) is highly mobile and can be transported into the edible tissues of crop plants (Pan et al., 2019). To ensure food safety, sustainable technologies are urgently needed to prevent $\mathrm{Cd}$ accumulation in plants grown under $\mathrm{Cd}$-polluted conditions. However, the use of physical and chemical methods to remedy Cd-contaminated soil 
is impracticable, rendering these methods ineffective for implementation at field sites (Pulford and Watson, 2003). Mounting evidence has indicated that the exploitation of soilborne bacteria is an emerging alternative for preventing $\mathrm{Cd}$ uptake in plants (Ravanbakhsh et al., 2019; Zhou et al., 2019a; Tanwir et al., 2021).

$\mathrm{Cd}$ is a toxic metal element for plants that shares chemical similarity with some essential elements such as calcium (Ca), zinc $(\mathrm{Zn})$, and iron $(\mathrm{Fe}) . \mathrm{Cd}$ can compete with other mineral nutrients to bind with several functional proteins, leading to the serious impairment of plant growth (Schützendübel and Polle, 2002). Cd is primarily transported into root cells through some plasma membrane-localized metal transporters such as ironregulated transporters (IRTs) and zinc/iron-regulated transporter proteins (ZIPs), which results in severe interferences with $\mathrm{Fe}$ uptake (Clemens, 2006). Previous studies have indicated that increased Fe supply or rhizospheric Fe availability effectively inhibits Cd uptake in plants, which is closely associated with the competition between $\mathrm{Fe}$ and $\mathrm{Cd}$ for several metal transporters (Wu et al., 2012; Sebastian and Prasad, 2016). Cd-stressed plants often exhibit typical chlorotic symptoms, similar to those occurring in Fe deficient plants (Chen et al., 2017). Cd stress can trigger Fe deficiency responses, which are accompanied by the up-regulation of $\mathrm{Fe}$ acquisition-associated genes including FIT, encoding a functional homolog of the bHLH transcription factor Fer, IRT1 encoding an iron-regulated transporter, and FRO2, encoding a putative ferric reduction oxidase (Lešková et al., 2017). Recently, nitric oxide (NO) has been demonstrated to govern Fe deficiency responses in different plant species (Buet et al., 2019). Cd stress can stimulate root NO burst and further initiate signaling pathways resembling those induced by Fe deficiency via the enhancement of IRT1 and FRO2 transcripts. Cd-induced IRT1 expression has also been implicated in the promotion of $\mathrm{Cd}$ absorption, thus aggravating Cd toxicity in Arabidopsis (Lei et al., 2020). Several phytohormones such as jasmonic acid (JA) and gibberellic acid (GA) negatively regulate the NO-dependent signaling pathways in Cd-stressed plants (Zhu et al., 2012; Lei et al., 2020). In Arabidopsis, JA can inhibit root uptake of Cd by reducing root $\mathrm{NO}$ accumulation and thus down-regulating IRT1 expression (Lei et al., 2020). GA has also been reported to repress the root NO burst and the transcription of $\mathrm{Cd}$ uptakerelated $I R T 1$, thereby attenuating Cd toxicity in plants (Zhu et al., 2012). Therefore, the inhibition of NO-mediated IRT1 expression in Cd-stressed plants is an alternative strategy for interdicting Cd absorption.

Plants coexist with a myriad of soil microbes that play fundamental roles in maintaining plant health and productivity (Cheng et al., 2019). The manipulation of soil-borne bacteria can potentially suppress disease incidence of plants (Liu et al., 2019), increase agricultural production (Gouda et al., 2018), lower the emissions of greenhouse gases (Wu et al., 2018), and reduce heavy metal contents within the tissues (Xu et al., 2018; Ravanbakhsh et al., 2019; Tanwir et al., 2021), leading to more sustainable agricultural practices. A plant host harbors a mass of bacterial species inside its tissues that impacts plant growth and health (Hardoim et al., 2015).
It is well documented that endophytic bacteria can colonize the roots and activate a series of sophisticated mechanisms for aiding plant adaptation to harmful conditions (Lugtenberg and Kamilova, 2009). Several endophytic bacteria can improve plant growth and survival under adverse stresses via the modulation of hormone-related signaling pathways (Oleńska et al., 2020). Mounting evidence has indicated that microbemediated changes in hormonal status in plants are primarily attributable to microbial synthesis (e.g., abscisic acid and auxin), degradation (1-aminocyclopropane-1-carboxylate deaminasemediated ethylene metabolism) of hormones, and alterations in hormone metabolism by bacterial volatile compounds (Bal et al., 2013; Sharifi and Ryu, 2018; Xu et al., 2018). However, it thus far remains unclear how the endophytederived signals control the host hormone metabolic pathways for alleviating Cd toxicity.

In this study, we explored the impacts of root endophytic bacteria on tomato adaptation to Cd stress. Among these bacterial isolates, the acetic acid-producing bacteria Lysinibacillus fusiformis greatly elevated the capability of the tomato plants to ameliorate $\mathrm{Cd}$ toxicity. Transcriptomic, elemental and pharmacological analyses were further combined to elucidate the mechanisms of the endophyte-mediated detoxification of $\mathrm{Cd}$ in plants. We found that the interactions between $L$. fusiformis and the tomato roots activated JA signaling pathways to repress the entry of $\mathrm{Cd}$ into the roots. Therefore, our study provided a new avenue for exploiting acetic acid-producing endophytes to steer host JA signaling pathways for impeding Cd uptake.

\section{MATERIALS AND METHODS}

\section{Isolation of Endophytic Bacteria From Cd-Treated Tomato Roots}

Root samples were harvested from 2 months-old tomato plants cultivated in Cd-polluted soils (100 $\mathrm{mg} \mathrm{Cd} \mathrm{kg}^{-1}$ soil). Approximately $1.0 \mathrm{~g}$ of roots was placed into $50 \mathrm{~mL}$ of plastic tube with sterile water for ultrasonic cleaning for $15 \mathrm{~min}$, followed by sterilization with $1 \% \mathrm{NaClO}$ for $5 \mathrm{~min}, 75 \%$ alcohol for 2 min and rinsing five times with sterile water. The nutrient agar (NA) plates coated by the last rinsed water were used as a control. The sterilized roots were ground with $15 \mathrm{~mL}$ of $0.2 \mathrm{M}$ phosphate buffered saline (PBS) solution and allowed to stand for $15 \mathrm{~min}$. After that, $1 \mathrm{~mL}$ of the supernatant was serially diluted and spread on the NA agar plates containing $20 \mathrm{mg} \mathrm{L}^{-1} \mathrm{CdCl}_{2}$ for $72 \mathrm{~h}$. Bacterial colonies were randomly picked and purified, and a total of 36 isolates were obtained. Bacterial genomic DNA was extracted for amplifying and sequencing $16 \mathrm{~S}$ rRNA genes.

To evaluate the effects of bacterial isolates on the Cd-stressed tomato plants, a high-throughput screening test was designed. Briefly, tomato seeds were sterilized with $1 \% \mathrm{NaClO}$ for $10 \mathrm{~min}$ and then washed with sterile water. Subsequently, these seeds were cultured on 1/2 Hoagland's medium for 10 days (d) and then placed on $0.6 \%$ agar plates. Each root was incubated with $20 \mu \mathrm{L}$ of bacterial inoculum at $5 \times 10^{7} \mathrm{CFU} \mathrm{mL} \mathrm{m}^{-1}$ for $48 \mathrm{~h}$ at $25^{\circ} \mathrm{C}$ in the dark, and then transferred to the soil 
(pH 6.5, organic matter $16.7 \mathrm{~g} \mathrm{~kg}^{-1}$, total $\mathrm{N} 1.25 \mathrm{~g} \mathrm{~kg}^{-1}$, available P $8.9 \mathrm{mg} \mathrm{kg}^{-1}$; available $\mathrm{K} 102.2 \mathrm{mg} \mathrm{kg}^{-1}$ ) under a photoperiod of light $(16 \mathrm{~h}) /$ dark $(8 \mathrm{~h})$ at $25^{\circ} \mathrm{C}$. Before transplantation, the soil was saturated with the solution of $\mathrm{CdCl}_{2}$ to achieve $100 \mathrm{mg} \mathrm{Cd} \mathrm{kg}{ }^{-1}$ soil for 2 months and was then autoclaved at $120^{\circ} \mathrm{C}$ for $1 \mathrm{~h}$ before use. The values of maximal PSII photochemical efficiency (Fv/Fm), soil plant analysis development (SPAD), and shoot fresh weight (SFW) were measured for assessing the $\mathrm{Cd}$ resistance of plants after 2 weeks of exposure to Cd stress.

\section{Qualitative Analyses of Acetic Acid-Producing Bacteria, Ultraviolet Mutagenesis and Measurement of Root Acetic Acid Levels and Rhizospheric Organic Acids}

Bacterial strains were cultured in basic medium $(\mathrm{pH} 6.8 ; 1 \%$ yeast extract, $1 \%$ glucose, and $3 \%$ ethanol) at $30^{\circ} \mathrm{C}$ for $96 \mathrm{~h}$, followed by centrifugation at $8,000 \times \mathrm{g}$ for $15 \mathrm{~min}$. Subsequently, $5 \mathrm{~mL}$ of the supernatant was neutralized with $0.1 \mathrm{M} \mathrm{NaOH}$, followed by addition of $20 \mu \mathrm{L}$ of $5 \% \mathrm{FeCl}_{3}$. The mixture was heated for $10 \mathrm{~min}$ at $100^{\circ} \mathrm{C}$. The formation of reddish brown precipitates indicated the presence of acetic acid. To generate acetic acid-deficient strains, wild-type (WT) strains were mutated by ultraviolet radiation and then spread on the agar plates ( $1 \%$ yeast extract, $1 \%$ glucose, $2 \% \mathrm{CaCO}_{3}$, and $1.5 \%$ agar) at $28^{\circ} \mathrm{C}$ for $48 \mathrm{~h}$. The mutated strains that did not produce a transparent zone indicated an inability to produce acetic acid for dissolving $\mathrm{CaCO}_{3}$. Furthermore, to measure the acetic acid content, $1.0 \mathrm{~g}$ of root tissue was homogenized and centrifuged at $12,000 \times \mathrm{g}$ for $15 \mathrm{~min}$ at $4^{\circ} \mathrm{C}$. Then, acetone was used to dilute the supernatant for acetic acid quantification by gas chromatography-mass spectrometry (GC-MS) (Kim et al., 2017). In addition, root-released organic acids were detected using high performance liquid chromatography (HPLC) as reported by Pii et al. (2015).

\section{Pot and Split-Root Experiments}

For the pot experiments, 10 -d-old tomato roots were incubated with $20 \mu \mathrm{L}$ of cell suspension of L. fusiformis $\mathrm{Cr} 33$ at $5 \times 10^{7} \mathrm{CFU}$ $\mathrm{mL}^{-1}$ as described above. These plants were then transplanted to plastic pots filled with the Cd-polluted soil $(100 \mathrm{mg} \mathrm{Cd} \mathrm{kg}-1$ soil). To conduct split-root assays, 3 -weeks-old tomato roots were placed into split-root boxes containing 1/2 Hoagland's medium as reported by Zhou et al. (2019a). In split-root systems, left side of each root box was supplied with bacterial suspensions, while the other side was not. After $3 \mathrm{~d}$ of culture, these plants were

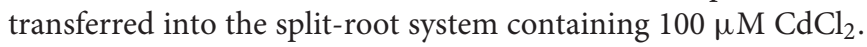
The medium in the root box was replaced every $3 \mathrm{~d}$. In addition, the root colonization of $L$. fusiformis $\mathrm{Cr} 33$ was quantified by quantitative real-time PCR (qRT-PCR) with a pair of genespecific primers for amplifying the $16 \mathrm{~S}$ rRNA gene fragment of L. fusiformis (F: 5'-ACGGTTTCGGCTGTCGCTAT-3'; R: 5' TTCCCTACTGCTGCCTCCC-3').

\section{Determination of Metal Content and in situ Localization of Cd}

To measure the total metal content, plant tissues were washed and dried for $24 \mathrm{~h}$ at $80^{\circ} \mathrm{C}$, followed by treatment with $\mathrm{HNO}_{3} / \mathrm{HClO}_{4}(4: 1, \mathrm{v} / \mathrm{v})$ and dilution with deionized water as described previously by Lei et al. (2014). The soluble Fe content was detected according to Cassin et al. (2009). Briefly, plant tissues were ground with deionized water and then centrifuged at $12,000 \times \mathrm{g}$ for $15 \mathrm{~min}$. The supernatant was used to measure soluble Fe content. The contents of total $\mathrm{Fe}$ and $\mathrm{Cd}$, and soluble Fe were quantified via inductively coupled plasma-atomic emission spectroscopy (ICP-AES). For measuring apoplastic Fe content, the roots were serially treated with $\mathrm{CaSO}_{4}, 2$.2-bipyridyl, $\mathrm{N}_{2}$ and $\mathrm{Na}_{2} \mathrm{~S}_{2} \mathrm{O}_{4}$ according to Jin et al. (2007). The collected solutions were used to determine root apoplastic Fe via detection of the $\mathrm{Fe}^{2+}$-bipyridyl complex at $520 \mathrm{~nm}$.

In situ localization of $\mathrm{Cd}$ was detected in the roots as described by Balestri et al. (2014). Briefly, excised roots were rinsed with deionized water and then immediately immersed in dithizone working solution containing $30 \mathrm{mg}$ dithizone, $20 \mathrm{~mL}$ deionized water and $60 \mathrm{~mL}$ acetone for $2 \mathrm{~h}$. The roots with reddish precipitates were washed with deionized water and then photographed. Additionally, the availability of $\mathrm{Fe}$ and $\mathrm{Cd}$ in the rhizospheric soil was extracted and analyzed by ICP-AES according to Chen et al. (2015).

\section{RNA Sequencing (RNA-Seq) and qRT-PCR Analyses}

Three-week-old tomato plants grown in hydroponic systems were subjected to treatment with or without bacterial suspension for $48 \mathrm{~h}$. These plants were then transferred into the splitroot systems containing $100 \mu \mathrm{M} \mathrm{CdCl}$. After that, the root tissues were harvested and immediately ground in liquid nitrogen. Then, total RNA from the root samples was extracted using TRIzol reagent (Invitrogen, United States) according to the manufacturer's instructions. The RNA samples from three biological repeats were analyzed using an Agilent 2100 Bioanalyzer and then used for RNA sequencing through the Illumina HiSeq 4000 platform (Illumina, United States). Clean reads were mapped to the reference genome sequence of Solanum lycopersicum Heinz 1706, and then submitted to the NCBI SRA database (No. PRJNA695320). The R package DEGseq was used to screen differentially expressed genes (DEGs) at $P \leq 0.05$ and a value of $\log _{2}$ fold change $>1.0$ or $<-1.0$. Gene Ontology (GO) enrichment analyses of the DEGs were screened at a cutoff of FDR $\leq 0.05$ using the GOseq R package (Chen et al., 2005). Kyoto Encyclopedia of Genes and Genomes (KEGG) pathway analyses were conducted using the KOBAS software (Mao et al., 2005).

For the qRT-PCR analyses, total RNA samples were extracted and used as qPCR templates. The qRT-PCR reactions were performed using the SYBR Green qPCR Master Mix (Takara, Japan) in an Applied Biosystems ${ }^{\text {TM }} 7500$ Real-Time PCR system. The tomato Actin gene was used as an internal control. The primers used for qRT-PCR analyses were reported recently by Zhou et al. (2019b). 


\section{Detection of Root Ferric Chelate Reductase (FCR) Activity and 2,3,5-Triphenyltetrazolium Chloride (TTC) Assays}

Root FCR activities were measured as described by Grusak (1995). Briefly, whole roots were incubated in the assay solution (0.1 mM Fe-EDTA, 0.1 mM FerroZine, $0.5 \mathrm{mM} \mathrm{CaSO}_{4}, 0.1 \mathrm{mM}$ bathophenanthroline-disulfonic; $\mathrm{pH} 5.5$ ) for $2 \mathrm{~h}$ at $28^{\circ} \mathrm{C}$. Absorbance of the solutions was determined at $562 \mathrm{~nm}$ and the formation of $\mathrm{Fe}^{2+}$-FerroZine was detected using an extinction coefficient of $27.9 \mathrm{~mm}^{-1} \mathrm{~cm}^{-1}$. To measure cell viability, tomato roots were incubated in $15 \mathrm{~mL}$ of assay solution $(0.4 \%$ TTC in $60 \mathrm{mM}$ PBS, $\mathrm{pH} \mathrm{7.0)}$ for $6 \mathrm{~h}$ at $37^{\circ} \mathrm{C}$. Reduced TTC was extracted with ethanol and the absorbance was recorded at 485 nm (Hawrylak-Nowak et al., 2015).

\section{Analyses of Physiological Indexes, Root JA and NO Content and Ultrastructural Observation}

Chlorophyll content was assessed on the fully expanded leaves based on the analysis of the SPAD values using a portable chlorophyll meter as previously reported by Lei et al. (2014). The values of Fv/Fm and actual PSII photochemical efficiency (ФPSII) were determined using a FluorCam 7 system (Zhou et al., 2019a). The chlorophyll levels were analyzed based on the method described by Porra (2002). Briefly, leaf tissues were immersed in $80 \%$ acetone and incubated for $2 \mathrm{~d}$ in the dark. Absorbance of the extracted solutions was measured at 645 and $663 \mathrm{~nm}$. The levels of chlorophyll were assessed using the formula: $20.21 \times \mathrm{A} 645+8.02 \times$ A663. In addition, $\mathrm{H}_{2} \mathrm{O}_{2}$ content, electrolyte leakage (EL), and malondialdehyde (MDA) values were measured as described by Wang et al. (2010). Root JA content was measured as reported by Kim et al. (2017). Briefly, about $200 \mathrm{mg}$ of fresh tomato roots were ground into the powder using liquid nitrogen and then combined with $2 \mathrm{~mL}$ of extraction solution (methanol: formic acid:water = 15:1: 4), followed by centrifugation at $12,000 \times \mathrm{g}$ for $30 \mathrm{~min}$. The supernatant was loading into a Sep-Pak C18 column and then eluted with the extracted solution. The collected solution was evaporated and then dissolved in $200 \mu \mathrm{L}$ of $80 \%$ methanol for analyzing JA content using HPLC. In addition, root NO content was quantified by the oxyhemoglobin-based spectrophotometric assay as described recently by Zhou et al. (2019b). To observe chloroplast ultrastructure, leaf tissues were cut into small pieces and immediately fixed with $1 \%$ glutaraldehyde, and then subjected to gradient dehydration with acetone. Finally, the leaf samples were embedded in EPON 812 resin and cut into sections (70-100 nm) for ultrastructural observations.

\section{Statistical Analysis}

The experimental data were analyzed by SPSS 10.0 software (SPSS Inc., Chicago, United States) with using Student's $t$-test or Duncan's multiple range tests with one-way analysis of variance at $P<0.05$.

\section{RESULTS}

\section{Screening of Root Endophytic Bacteria With Cd-Detoxifying Properties in Plants}

A total of 36 endophytic bacteria strains were isolated from the roots of 2-month-old tomato plants cultivated in soil polluted with about $100 \mathrm{mg} \mathrm{Cd} \mathrm{kg}^{-1}$ soil. Bacterial isolates were identified by PCR amplification of $16 \mathrm{~S}$ rRNA genes, and the sequences were assigned to species using the NCBI blast tool (Supplementary Table 1). Based on $97 \%$ sequence similarity for the 16S rRNA genes, these bacterial strains were assigned to six phylogenetic taxa at the class level, including Alpha-, Beta- and Gamma-proteobacteria, Bacilli, Corynebacteriales, and Flavobacteria (Figure 1A). Furthermore, these isolates were selected to assess their potential for detoxifying $\mathrm{Cd}$ in the tomato plants. Using high-throughput screening assays (Figure 1B), nine bacterial strains were found to distinctly mitigate $\mathrm{Cd}$ stress in the tomato plants, as reflected by the higher values of $\mathrm{Fv} / \mathrm{Fm}$, SPAD and SFW, which were used as a proxy assessment for Cd toxicity in plants (Figure 1C). Compared with the noninoculated (control) plants, soil drenched with the nine isolates significantly reduced shoot $\mathrm{Cd}$ content, whereas some of the isolates increased the root $\mathrm{Cd}$ content (Figures 1D,E). Among these isolates, L. fusiformis $\mathrm{Cr} 33$ exhibited the greatest ability to relieve $\mathrm{Cd}$ stress and inhibit plant uptake of $\mathrm{Cd}$, and was thus selected for the subsequent experiments.

\section{Inoculation With $L$. fusiformis Alleviated Cd Stress and Reduced Cd Uptake in Tomato}

After 4 weeks of Cd treatment, serious chlorosis was observed in new leaves with a marked reduction in chloropyll content and Fv/Fm values, but this was clearly relieved by supplying the soil with L. fusiformis Cr33 (Supplementary Figures 1AC). The Cd content was notably lower in the shoots and roots of the inoculated plants than the non-inoculated (control) plants (Supplementary Figure 1D). Within 4 weeks of inoculation, the population of L. fusiformis $\mathrm{Cr} 33$ had abundantly colonized the roots within $7 \mathrm{~d}\left(0.3-1.2 \times 10^{7} \mathrm{CFU} \mathrm{g}^{-1}\right)$, following which it decreased $\left(0.4-3.5 \times 10^{6} \mathrm{CFU} \mathrm{g}^{-1}\right)$ (Supplementary Figure 1E).

In the split-root systems, 3-weeks-old tomato roots were treated with or without cell suspension of Cr33 (Figure 2A). $\mathrm{Cd}$ stress resulted in chlorotic symptoms with a reduction in chlorophyll levels and biomass (Figures 2B-F). However, root inoculation with $\mathrm{Cr} 33$ inhibited root growth compared with the non-inoculated (control) plants under $\mathrm{Cd}$ stress conditions (Figures 2C,F). The levels of $\mathrm{H}_{2} \mathrm{O}_{2}$ were distinctly increased in the leaves of the Cd-treated plants, whereas the Cr33-inoculated leaves accumulated less $\mathrm{H}_{2} \mathrm{O}_{2}$ levels than the control plants (Figure 2G). Accordingly, the inoculated plants displayed lower the values of MDA and EL in the leaves than the control plants (Figures 2H,I). Additionally, $\mathrm{Cd}$ exposure led to marked decreases in the values of Fv/Fm and ФPSII, whereas their values were remarkably lower in the control plants than the inoculated plants under $\mathrm{Cd}$ stress conditions (Figures 3A-C). However, there was no obvious 


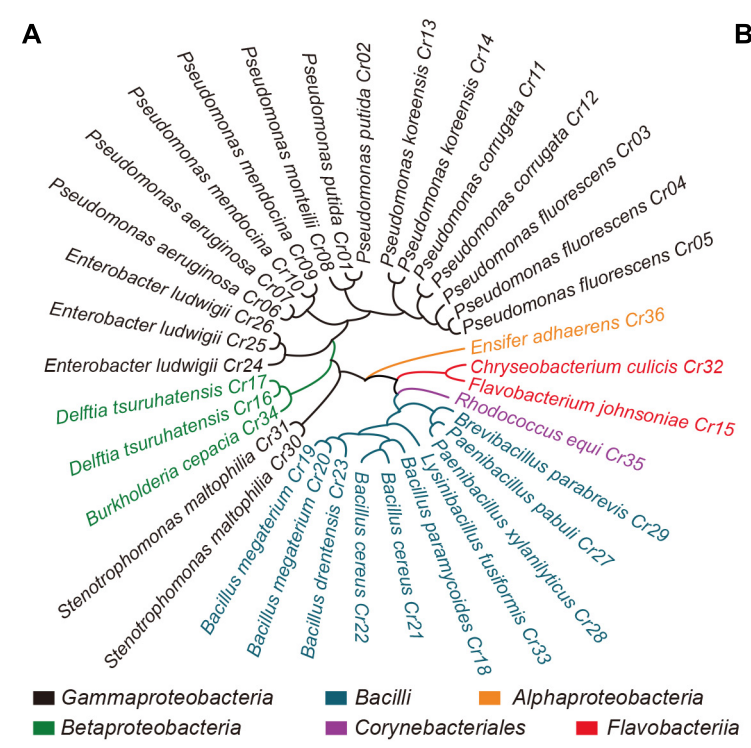

B

- Betaproteobacteria Corynebacteriales Flavobacteria

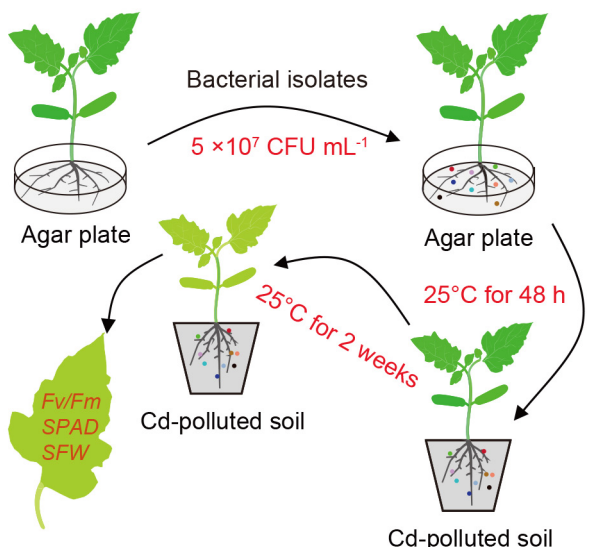

0

C

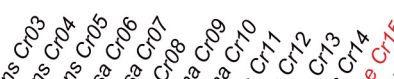

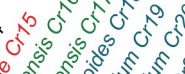

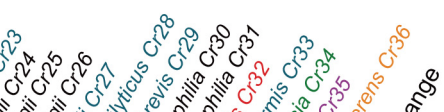

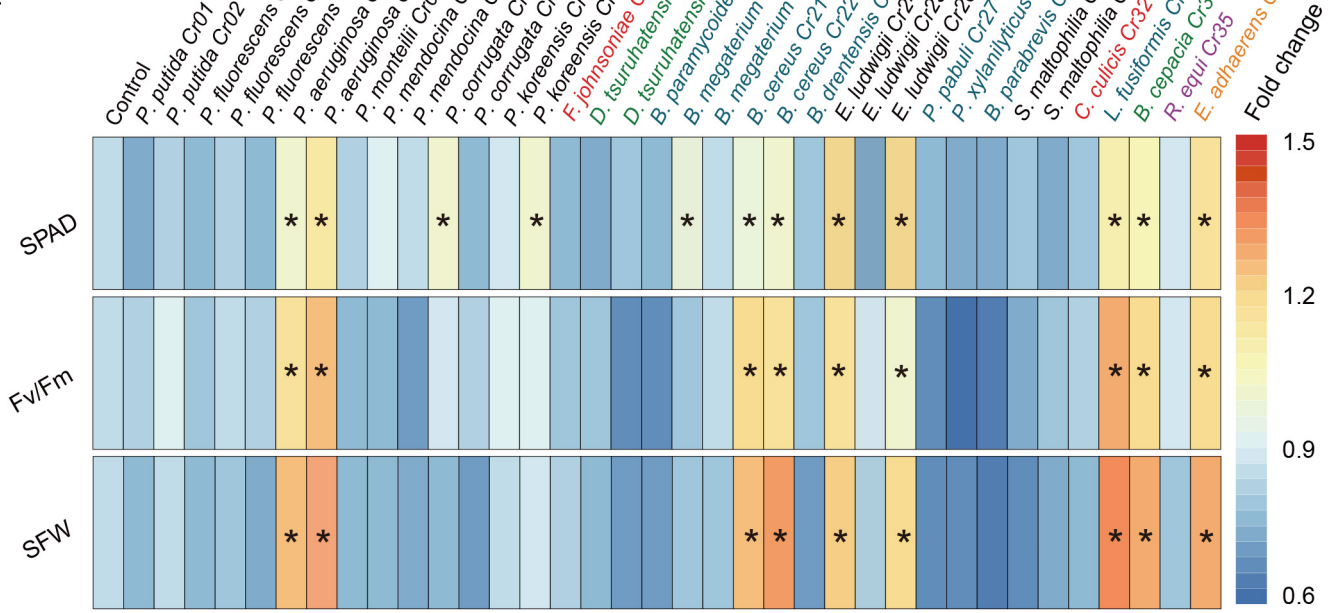

D
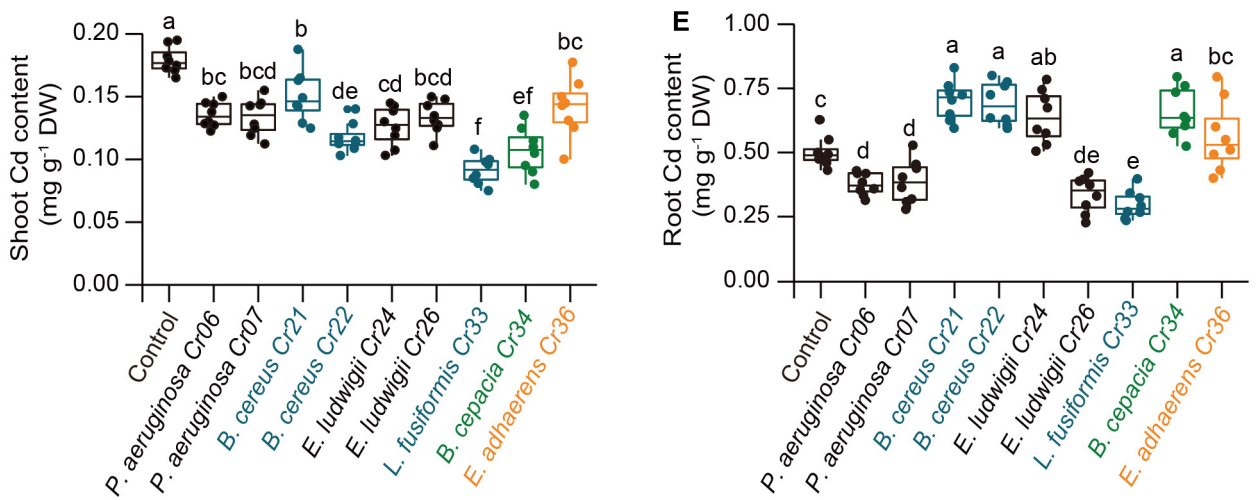

FIGURE 1 | Effects of soil drench with endophytic bacteria on Cd resistance in tomato plants. (A) Taxonomic cladogram of bacterial isolates. (B) High-throughput assays of $\mathrm{Cd}$-detoxifying bacterial isolates. Ten-day-old tomato roots were co-cultured with or without bacterial suspension on agar plate at $25^{\circ} \mathrm{C}$ in the dark for $48 \mathrm{~h}$. the non-inoculated (control) and inoculated plants were then transplanted into Cd-contaminated soils ( $100 \mathrm{mg} \mathrm{Cd} \mathrm{kg}^{-1} \mathrm{soil}^{\text {a }}$ at $25^{\circ} \mathrm{C}$. After 2 weeks of culture, the values of Fv/Fm, SPAD and SFW were determined for assessing the ability of plants to tolerate Cd stress. (C) Heatmap analysis for Fv/Fm, SPAD and SFW. Asterisks indicated significant differences between the control and inoculated plants ( $n=8$ biological replicates) using Student's test at $p<0.05$. (D) Shoot and (E) root Cd content. Different letters indicated significant differences among different bacterial strain-inoculated plants ( $n=8$ biological replicates) using Duncan's multiple range test at $p<0.05$. 
difference in photosynthesis between the control and inoculated plants under non-Cd condition (Figures 3A-C). Cd stress also led to a marked reduction in chloroplast number, swollen chloroplasts and fewer granum lamellae, but this was largely alleviated by root inoculation with Cr33 (Figure 3D). The TTC cell viability assays also showed that the inoculated plants maintained higher root viability than the control plants under $\mathrm{Cd}$ stress conditions (Supplementary Figure 2). Moreover, the inoculated plants exhibited less $\mathrm{Cd}$ content than the control plants (Figure 3E). Accordingly, in situ localization of $\mathrm{Cd}$ showed reddish precipitates in the Cd-treated roots, whereas the inoculated roots exhibited less reddish precipitates than the control roots (Figure 3F). The inoculated plants exhibited a reduction of about $50 \%$ shoot $\mathrm{Fe}$ content under Cd stress compared with the control plants, but no striking difference was observed for root $\mathrm{Fe}$ content (Figure 3G). However, the inoculated roots exhibited less apoplastic $\mathrm{Fe}$ $(\mathrm{ApoFe})$ and higher soluble $\mathrm{Fe}(\mathrm{SoFe})$ levels than the control plants (Figure $3 \mathbf{H}$ ).

\section{L. fusiformis-Derived Acetic Acid Increased Cd Resistance in Tomato}

The bioavailability of $\mathrm{Cd}$ and $\mathrm{Fe}$ in the rhizosphere is positively related to $\mathrm{Cd}$ toxicity in plants (Xu et al., 2018; Wang et al., 2020). We therefore determined their content in rhizospheric soils from both the control and Cr33-inoculated plants. The content of rhizospheric Cd was slightly higher in the bacteriatreated soils than in the untreated soils (Figure 4A). By contrast, the levels of rhizospheric Fe were about twofold higher in the bacteria-treated soils than in the untreated soils. Root-secreted organic acids can influence the availability of nutrient elements and heavy metals in the rhizosphere (Rajkumar et al., 2012; Montiel-Rozas et al., 2016). Thus, the levels of organic acids in the rhizospheric soils were determined. Compared with the control plants, the rhizospheric soils from the inoculated plants had greater total organic acid content. Among these organic acids, the rhizospheric soils from the inoculated plants displayed significantly higher acetic acid levels than the control plants (Supplementary Figure 3). Moreover, qualitative assays revealed that the reaction of $\mathrm{Cr} 33$ culture with $\mathrm{FeCl}_{3}$ produced reddish brown substances (Supplementary Figure 4), indicating that L. fusiformis was an acetic acidproducing bacterium.

As shown in Figure 4B, two acetic acid-deficient mutants (mCr33-1 and -2) did not considerably increase the root acetic acid levels compared with Cr33. Moreover, the Cd-induced chlorotic symptoms and reduction of Fv/Fm values were not largely ameliorated by the mutant strains (Figures 4C,D). The levels of $\mathrm{Cd}$ were distinctly higher in the shoots and roots of the mCr33-exposed plants than those of the Cr33exposed plants (Figure 4E). In line with this, in situ localization of $\mathrm{Cd}$ showed a notable reduction in reddish precipitates in the roots colonized by $\mathrm{Cr} 33$, but not in the $\mathrm{mCr} 33$ inoculated roots (Figure 4F). To further confirm whether high-level acetic acid conferred increased $\mathrm{Cd}$ resistance in plants, split-root assays were conducted as described above.
Acetic acid (AA) treatment markedly alleviated the Cdinduced leaf chlorosis with higher Fv/Fm values and less $\mathrm{Cd}$ content (Figures 4C-E). Consistent with this, the reddish precipitates were substantially reduced in the AA-treated roots (Figure 4F).

\section{Transcriptome Analyses of $L$. fusiformis-Treated Tomato Roots}

To probe the molecular mechanisms of L. fusiformis-induced Cd resistance of tomato plants, RNA-Seq was performed to examine gene expression profiles of the Cd-treated roots colonized by Cr33. For this, tomato plants cultured in hydroponic systems with or without $\mathrm{Cr} 33$ were treated with $100 \mu \mathrm{M} \mathrm{CdCl} 2$ for 0 and $48 \mathrm{~h}$ (Figure 5A). Gene expression changes in the roots were examined by comparing the control plants (Cd48) with the Cr33-inoculated plants (Cr33 + Cd48). An additional condition evaluation of the impacts of $100 \mu \mathrm{M} \mathrm{Cd}^{2+}$ on the gene expression profiles in the roots was also conducted. A total of 1,015 (Supplementary Table 2) and 863 (Supplementary Table 3). DEGs exhibited significant differential expression in both Group I (Cd48 vs. -Cd) and II (Cr33 + Cd48 vs. Cd48), respectively (Figures 5B,C). The GO enrichment analyses of the DEGs showed that several genes involved in diverse processes such as detoxification and response to stimulus were remarkably induced by $\mathrm{Cd}$ stress and Cr33 (Supplementary Figure 5). As shown in Figure 5D, KEGG enrichment pathway analyses for the Group I revealed that Cd stress strikingly impacted several metabolic pathways such as sulfur and glutathione, which are responsible for the detoxification of $\mathrm{Cd}$ in plants (Gill and Tuteja, 2011; Han et al., 2020; Xu et al., 2020). Conversely, root inoculation with Cr33 markedly affected several pathways such as phenylalanine metabolism, phenylpropanoid biosynthesis, hormone biosynthesis and signal transduction (Figure 5E).

As shown in Figure 5C, 217 of the 518 DEGs that were greatly induced by $\mathrm{Cd}$ stress were down-regulated in the Cr33-inoculated roots, indicating that a mass of Cdresponsive genes in the roots was significantly repressed by Cr33 (Supplementary Table 4). Previously, the Cd-induced expression of Fe uptake-associated genes was shown to promote Cd uptake and enhance Cd toxicity in Arabidopsis plants (Figure 5F). Among the shared DEGs, the transcription levels of Fe uptake-associated genes including Fer, FRO1, and IRT1 were considerably increased in the Cd-exposed roots, whereas root inoculation with $\mathrm{Cr} 33$ significantly down-regulated their expression (Figure 5G). Additionally, several genes associated with the biosynthesis of JA and signal transduction were observably activated in the Cd-treated roots colonized by $\mathrm{Cr} 33$ (Supplementary Table 5).

\section{L. fusiformis Inhibited Root NO Burst and Fe Deficiency Response Under Cd Stress}

As shown in Figure 6A, Cd stress triggered the production of $\mathrm{NO}$ in the roots, whereas root NO burst was dramatically restrained in the Cr33-inoculated roots. Since NO is essential for activating the expression of the FER, IRT1, and FRO1 genes 

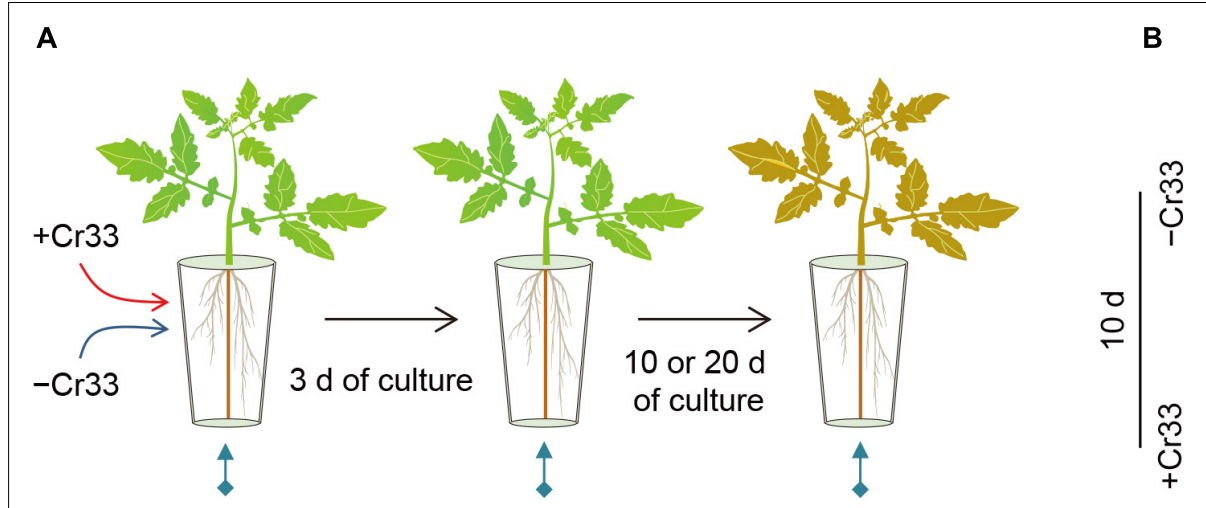

1/2 Hogland medium $1 / 2$ Hogland medium $1 / 2$ Hogland medium

$$
+0 \mu \mathrm{M} \mathrm{Cd} \quad+100 \mu \mathrm{M} \mathrm{Cd} \quad+100 \mu \mathrm{M} \mathrm{Cd}
$$

C
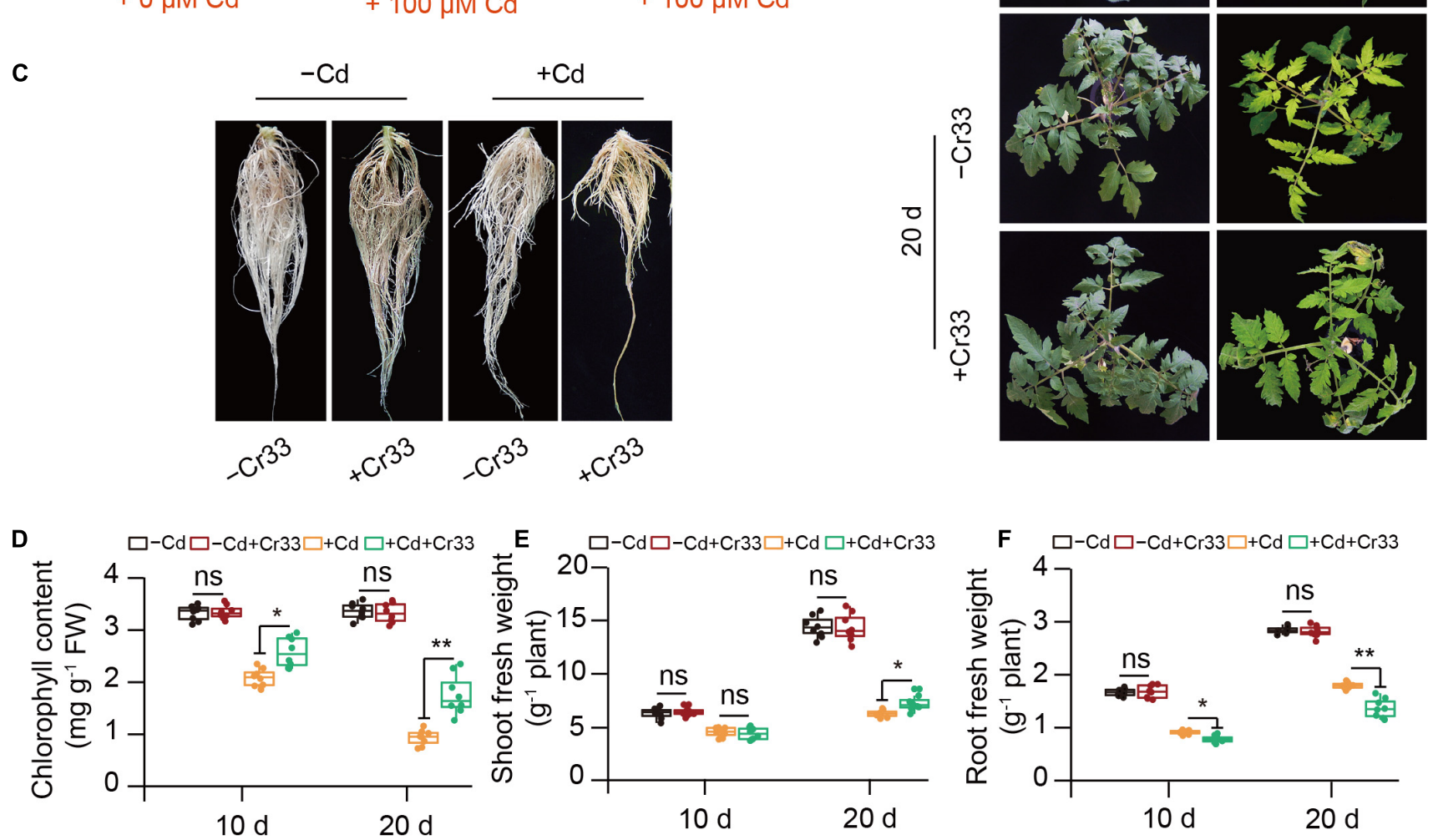

G
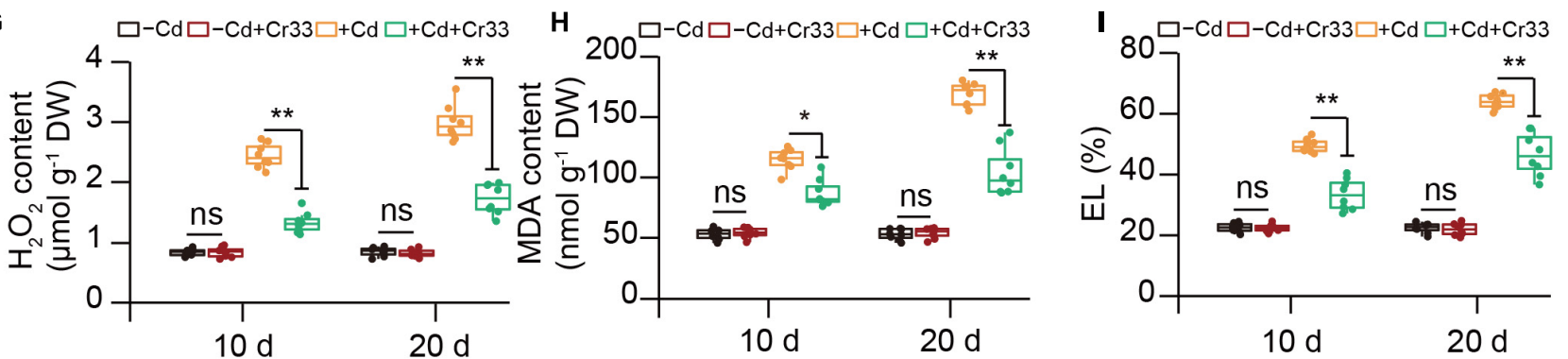

FIGURE 2 | Root inoculation with L. fusiformis Cr33 ameliorated Cd stress in tomato plants. (A) Three-weeks-old tomato plants cultured in the split-root systems were inoculated with or without Cr33 for $3 \mathrm{~d}$. Then, these plants were exposed to 0 or $100 \mu \mathrm{M}$ Cd with or without Cr33 for 10 and $20 \mathrm{~d}$, respectively. These plants were used to analyze shoot phenotypes (B), root growth after $20 \mathrm{~d}$ of culture (C), chlorophyll content (D), shoot (E) and root (F) fresh weight, $\mathrm{H}_{2} \mathrm{O}_{2}$ content (G), and $\operatorname{MDA} \mathbf{( H )}$ and EL (I). Asterisks indicated significant differences between the control and inoculated plants ( $n=8$ biological replicates) using Student's test (ns, not significant; $\left.{ }^{*} p<0.05 ;{ }^{* *} p<0.01\right)$. 

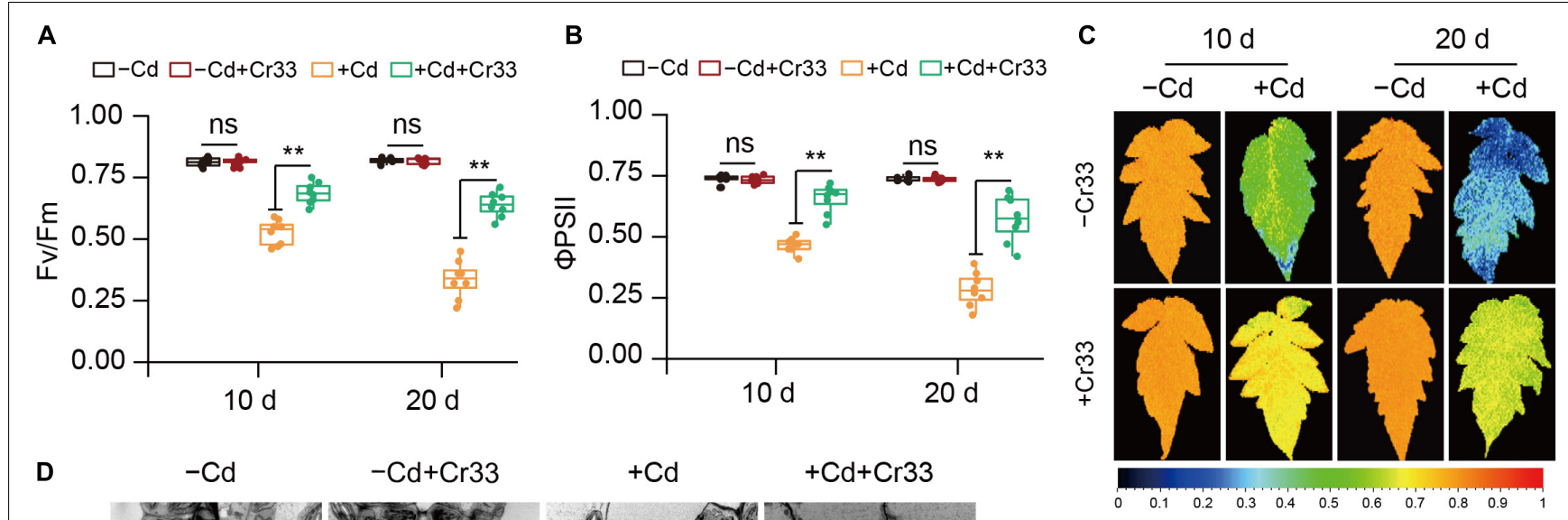

D

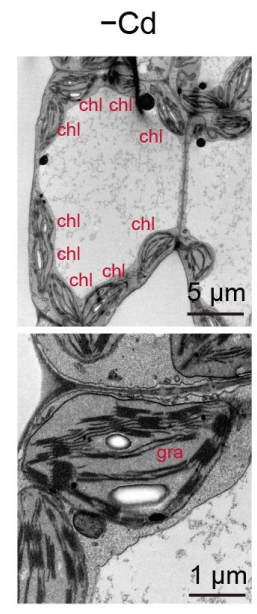

$-C d+C r 33$
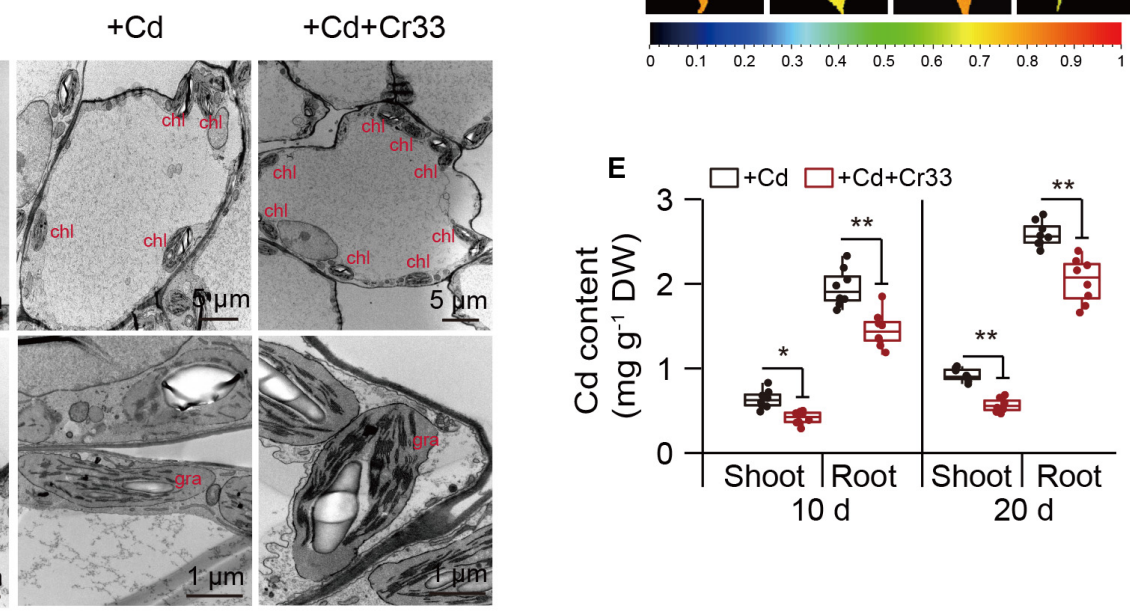

$\mathbf{F}$
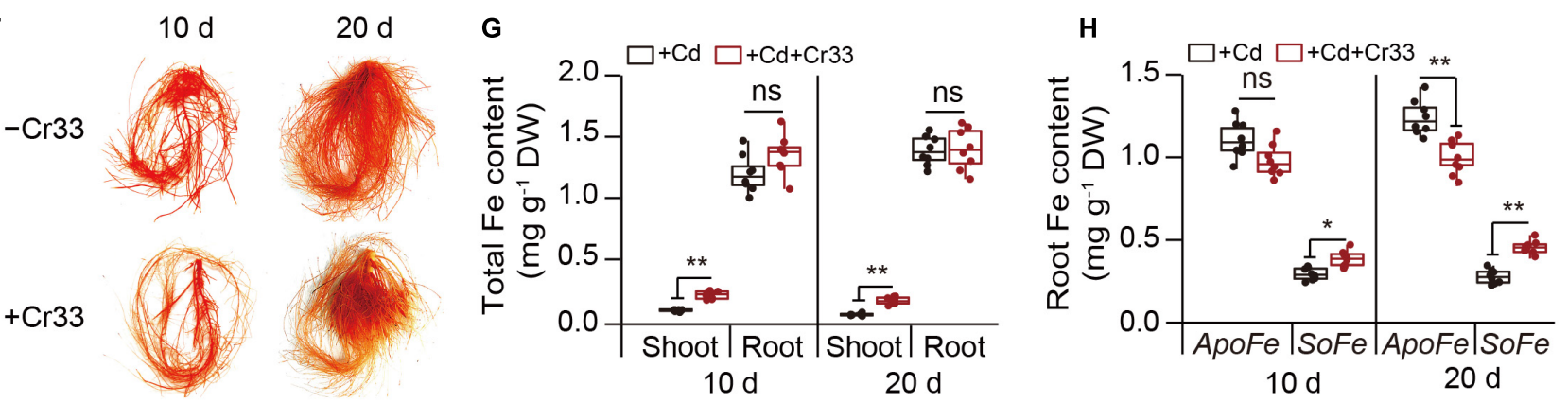

FIGURE 3 | Root inoculation with L. fusiformis Cr33 reduced Cd toxicity in tomato plants. Three-weeks-old tomato plants cultured in the split-root systems were inoculated with or without Cr33 for $3 \mathrm{~d}$. Then, these plants were exposed to 0 or $100 \mu \mathrm{M}$ Cd with or without Cr33 for 10 and $20 \mathrm{~d}$, respectively. These plants were used to analyze Fv/Fm (A), ФPSII (B), Fv/Fm images (C), chloroplast ultrastructure (chl, chloroplast; gra, grana) (D), shoot and root Cd content (E), root Cd localization (F), total Fe content (G), root apoplastic (ApoFe) and soluble Fe ( $\mathrm{SoFe}) \mathbf{( H )}$. Asterisks indicated significant differences between the control and inoculated plants ( $n=8$ biological replicates) using Student's test (ns, not significant; ${ }^{*} p<0.05 ;{ }^{* \star} p<0.01$ ).

in tomato roots under Fe deficiency (Graziano and Lamattina, 2007; Chen et al., 2015), we evaluated the effects of Cr33 on root $\mathrm{Fe}$ deficiency responses in the $\mathrm{Cd}$-exposed plants. Their transcripts were quantified by qRT-PCR in the roots after $48 \mathrm{~h}$ of exposure to Cd stress. The expression of the FER, IRT1, and $F R O 1$ genes was relatively higher in the Cd-treated roots than the untreated roots, but root inoculation with $\mathrm{Cr} 33$ reduced their transcripts (Figures 6B-D). The FCR activities in the Cd-treated roots were remarkably induced by $\mathrm{Cd}$ stress, whereas inoculation with Cr33 strikingly repressed root FCR activities (Figures 6E,F).
This decline of FCR activities was in concert with the reduced expression of FRO1 (Figure 6D).

\section{JA Signals Were Required for the L. fusiformis-Alleviated Cd Toxicity of Tomato}

High-level acetic acid promotes de novo JA synthesis and further activates the JA signaling pathway for improving drought tolerance in plants (Kim et al., 2017). For this reason, we 


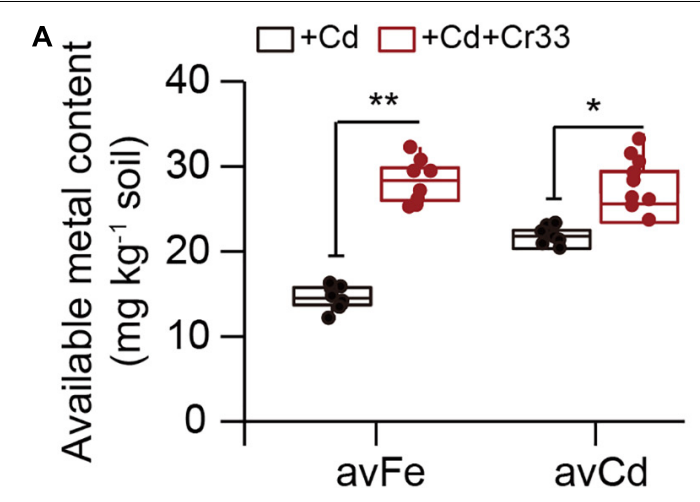

B

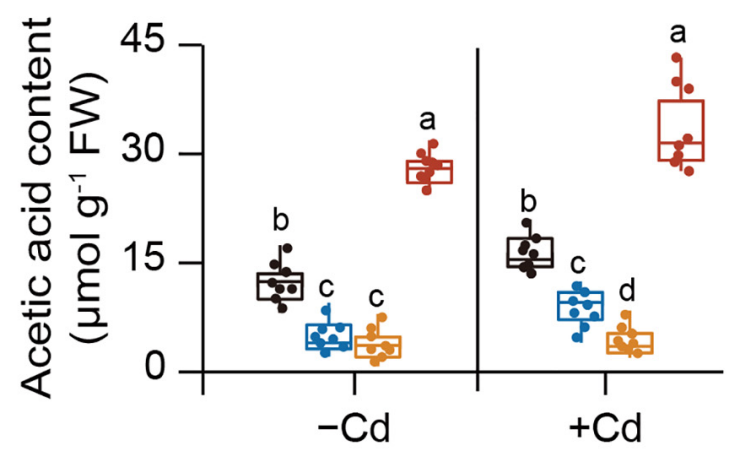

C

Cr33

mCr33-1

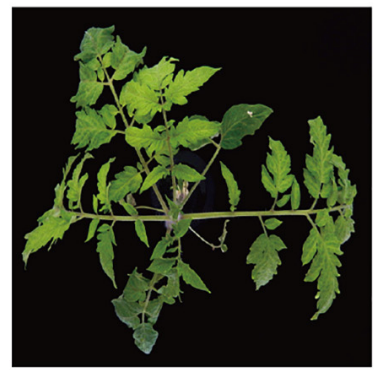

mCr33-2
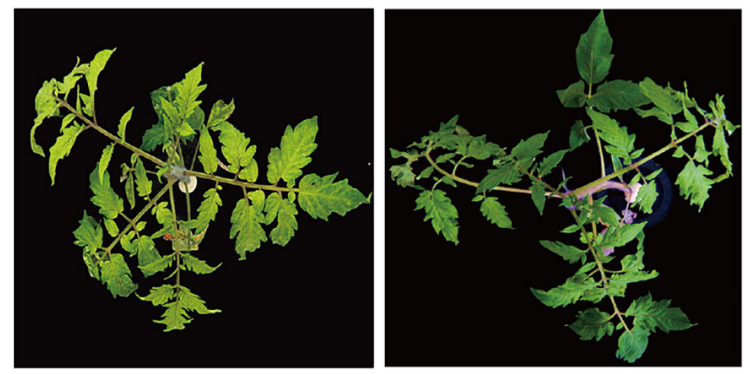

F

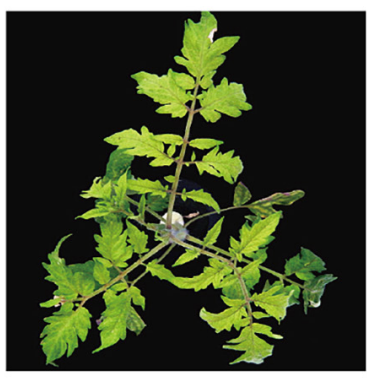

AA
Cr33

$\mathrm{mCr} 33-1$

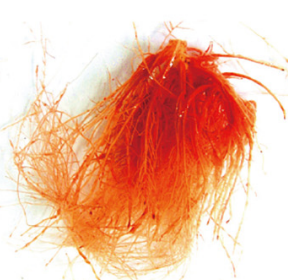

$\mathrm{mCr} 33-2$

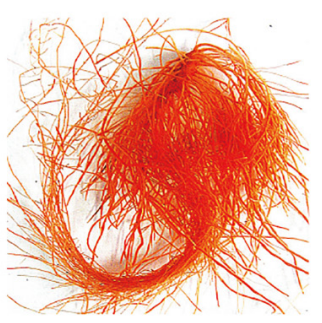

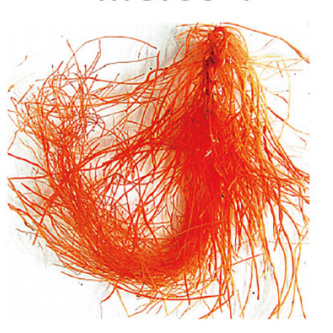

AA

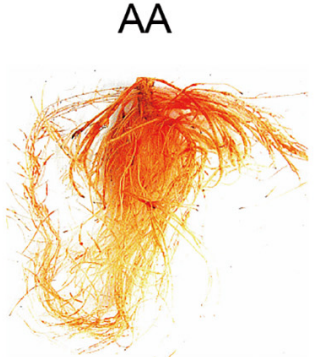

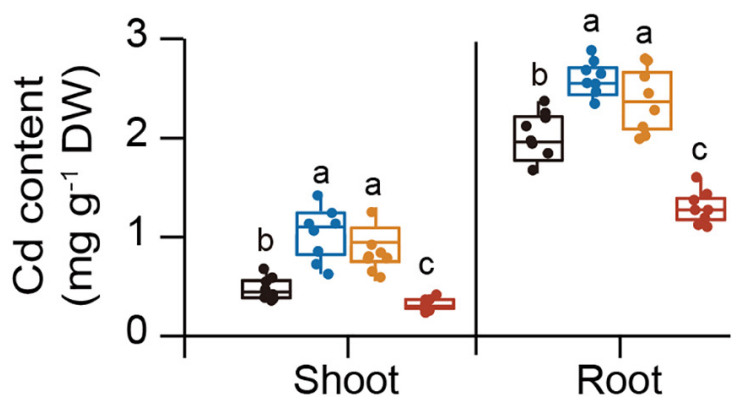

FIGURE 4 | L. fusiformis Cr33-released acetic acid conferred the increased availability of rhizospheric Fe and Cd, and Cd resistance in tomato plants. (A) The bioavailability of rhizospheric Fe and Cd was quantified after 2 weeks of soil drench with Cr33. Asterisks indicated significant differences between the non-inoculated (control) and inoculated plants ( $n=8$ biological replicates) using Student's $t$-test $\left({ }^{*} p<0.05 ;{ }^{* *} p<0.01\right)$. (B-F) Three-weeks-old tomato plants were cultured in the split-root systems containing $100 \mu \mathrm{M}$ Cd with or without Cr33 or its mutant strains (mCr33-1 and -2) for $20 \mathrm{~d}$. These plants were used to analyze root acetic acid content (B), shoot phenotypes (C), Fv/Fm images (D), shoot and root Cd content (E), and root Cd localization (F). Different letters indicated significant differences among different experimental groups ( $n=8$ biological replicates) using Duncan's multiple range test at $p<0.05$. 

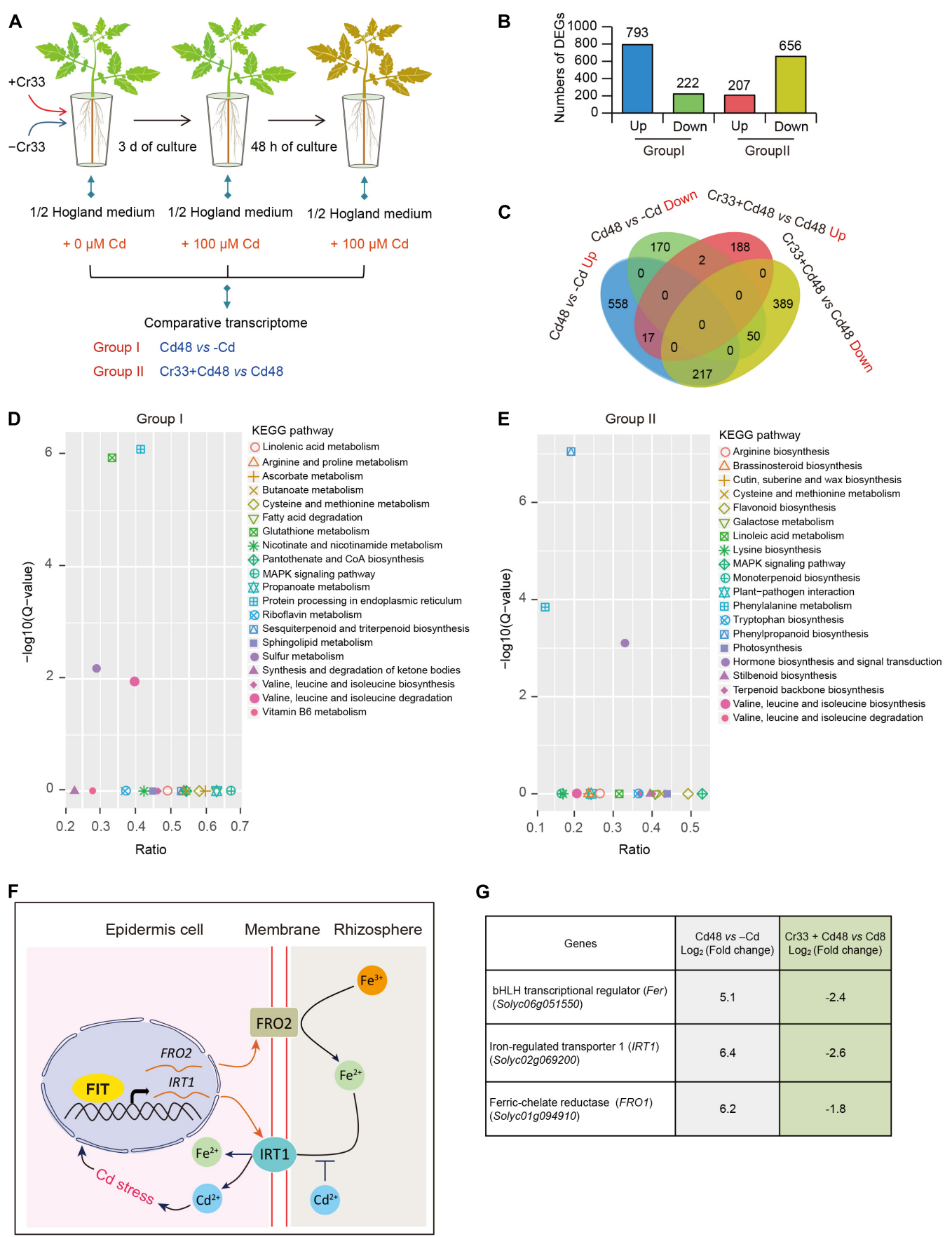

G

\begin{tabular}{|l|c|c|}
\hline \multicolumn{1}{|c|}{ Genes } & $\begin{array}{c}\text { Cd48 vs -Cd } \\
\text { Log }_{2} \text { (Fold change) }\end{array}$ & $\begin{array}{c}\text { Cr33 + Cd48 vs Cd8 } \\
\text { Log }_{2} \text { (Fold change) }\end{array}$ \\
\hline $\begin{array}{l}\text { bHLH transcriptional regulator (Fer) } \\
\text { (SolyC06g051550) }\end{array}$ & 5.1 & -2.4 \\
\hline $\begin{array}{l}\text { Iron-regulated transporter 1 (IRT1) } \\
\text { (Solyc02g069200) }\end{array}$ & 6.4 & -2.6 \\
\hline $\begin{array}{l}\text { Ferric-chelate reductase (FRO1) } \\
\text { (Solyc019094910) }\end{array}$ & 6.2 & -1.8 \\
\hline
\end{tabular}

FIGURE 5 | Transcriptome analyses of $L$. fusiformis Cr33-inoculated roots in response to Cd stress. (A) Three-week-old tomato plants cultured in the split-root systems were inoculated with or without $\mathrm{Cr} 33$ for $3 \mathrm{~d}$. Then, these plants were exposed to $100 \mu \mathrm{M}$ Cd with or without Cr33 for $48 \mathrm{~h}$. Total RNA samples extracted from the treated roots were used for RNA-Seq analyses, including Group I (Cd48 vs. -Cd) and II (Cr33 + Cd48 vs. Cd48). (B) Numbers of DEGs in both Group I and II. (C) Venna diagram of shared and specific DEGS between the Group I and II. (D,E) KEGG analyses for up-regulated DEGs from the Group I and II. (F) A model for the $\mathrm{Cd}$-activated Fe uptake leading to aggravate $\mathrm{Cd}$ toxicity in plants. Cd-induced expression of Fe uptake-related genes including FIT (a functional homolog of Fer), IRT1, and FRO2 resulted in the increased Cd uptake in plants. (G) Expression profiles of Fer, IRT1, and FRO1 in both the Group I and II.

assessed the effects of L. fusiformis Cr33 on the JA content in the Cd-exposed tomato roots. Compared with the non-inoculated (control) plants, the JA levels were remarkably increased in the Cr33-inoculated roots after $1 \mathrm{~d}$ of exposure to $\mathrm{Cd}$ stress and thereafter stabilized at a higher level over $7 \mathrm{~d}$ (Figure 7A). It was thus possible that the Cr33-induced increases in JA levels contributed to reducing the $\mathrm{Cd}$ toxicity in the plants. To validate this hypothesis, the Cd resistance of plants treated with methyl 

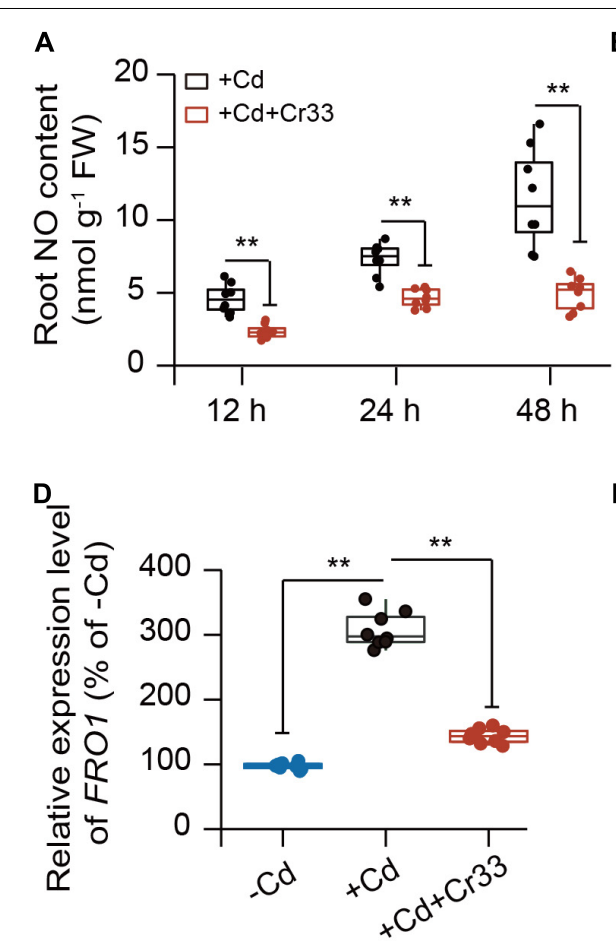
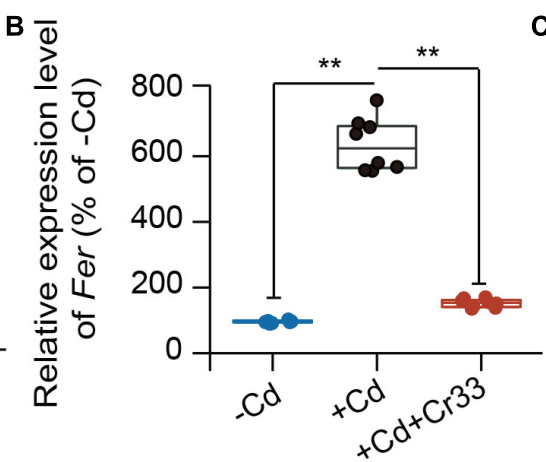

E

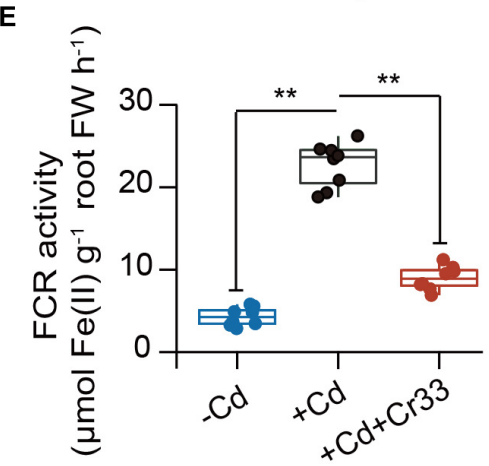

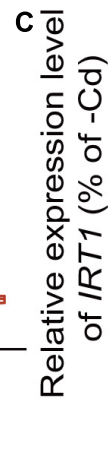

$\mathbf{F}$

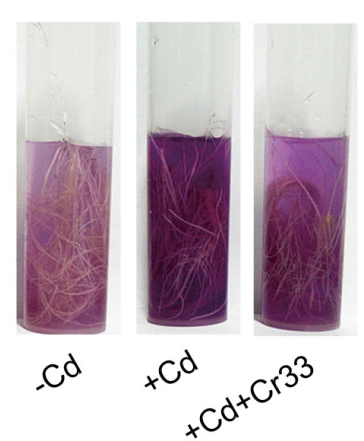

FIGURE 6 | Root inoculation with L. fusiformis Cr33 inhibited root Fe deficiency responses under Cd stress condition. Three-weeks-old tomato plants cultured in the split-root systems were inoculated with or without Cr33 for $3 \mathrm{~d}$. Then, these plants were exposed to $100 \mu \mathrm{M}$ Cd with or without Cr33 for $48 \mathrm{~h}$. These plants were used to analyze root No content (A), the expression of Fer (B), IRT1 (C), FRO1 (D). In addition, root FCR activities were quantified (E) and assessed by observing purple color (F). Asterisks indicated significant differences between different experimental groups $\left(n=8\right.$ biological replicates) using Student's $t$-test $\left({ }^{* *} p<0.01\right)$.

jasmonate (MeJA) was also evaluated. MeJA treatment strikingly relieved Cd stress in plants, as reflected by the higher chlorophyll content, $\mathrm{Fv} / \mathrm{Fm}$ values, and lower Cd levels (Figures 7B-F).

After $48 \mathrm{~h}$ of Cd treatment, root NO content was relatively lower in the MeJA-treated plants than the untreated (UT) plants, which was similar to the observation for the Cr33-inoculated roots (Figure 7G). Diethyldithiocarbamic acid (DIECA), a JA biosynthetic inhibitor, was applied to the Cd-treated plants colonized by $\mathrm{Cr} 33$. Root inoculation with $\mathrm{Cr} 33 \mathrm{did}$ not relieve $\mathrm{Cd}$ toxicity in the DIECA-treated plants, which displayed serious leaf chlorosis, lower chlorophyll levels and Fv/Fm values, and greater Cd content (Figures 7B-F). Similarly, DIECA treatment largely weakened the AA-alleviated Cd toxicity in the plants. Treatment with $\mathrm{Cr} 33$ or MeJA inhibited the transcription of Fer, FRO1 and IRT1, and the activities of FCR. Conversely, DIECA treatment enhanced root Fe deficiency responses in both the Cr33- and AAtreated plants (Figures $7 \mathbf{H}-\mathbf{K}$ ). In addition, the root NO content was remarkably increased in both the Cr33- and AA-treated plants when treated with DIECA (Figure 7G).

\section{DISCUSSION}

Remediating soil Cd contamination is costly and challenging, and thus there is an increasing demand for alternative strategies to address Cd pollution in agricultural soils (Pulford and Watson, 2003). Steering plant hormone metabolism toward the repression of Cd uptake may preserve food safety and improve plant performance under Cd-polluted conditions (Lei et al., 2017). Herein, we explored the impacts of the acetic acid-producing endophytic bacteria L. fusiformis on the plant hormone JA, which negatively regulates Fe uptake and translocation (Lei et al., 2020). The endophyte-derived acetic acid stimulated the JA biosynthesis and further suppressed the root Fe deficiency responses imposed by $\mathrm{Cd}$ stress, which contributed to reduction of $\mathrm{Cd}$ accumulation. Hence, our findings suggested that manipulation of host JA signals by root endophytic bacteria effectively prevented $\mathrm{Cd}$ absorption in the plants.

$\mathrm{Cd}$ stress often results in the reduction of plant photosynthesis, biomass and root viability (Hawrylak-Nowak et al., 2015; Xu et al., 2018; Pan et al., 2019). In accordance with previous reports on the harmful impacts of $\mathrm{Cd}$ stress, the values of photosynthetic parameters were largely decreased in the Cd-exposed leaves. Compared with the control plants, these photosynthetic indexes were observably higher in the L. fusiformis-inoculated plants under $\mathrm{Cd}$ stress. Observations of the photosynthetic apparatus further showed that the chloroplast structures were abnormal and that there were fewer chloroplasts and stacked grana in the Cd-exposed leaves. However, root inoculation with L. fusiformis mitigated the toxicity of $\mathrm{Cd}$ to the photosynthetic system. Cd stress also caused a considerable reduction in root viability, while the inoculated roots displayed higher viability than the control plants. Therefore, these findings indicated that the inoculated plants experienced fewer of the toxic effects imposed by $\mathrm{Cd}$ stress. 

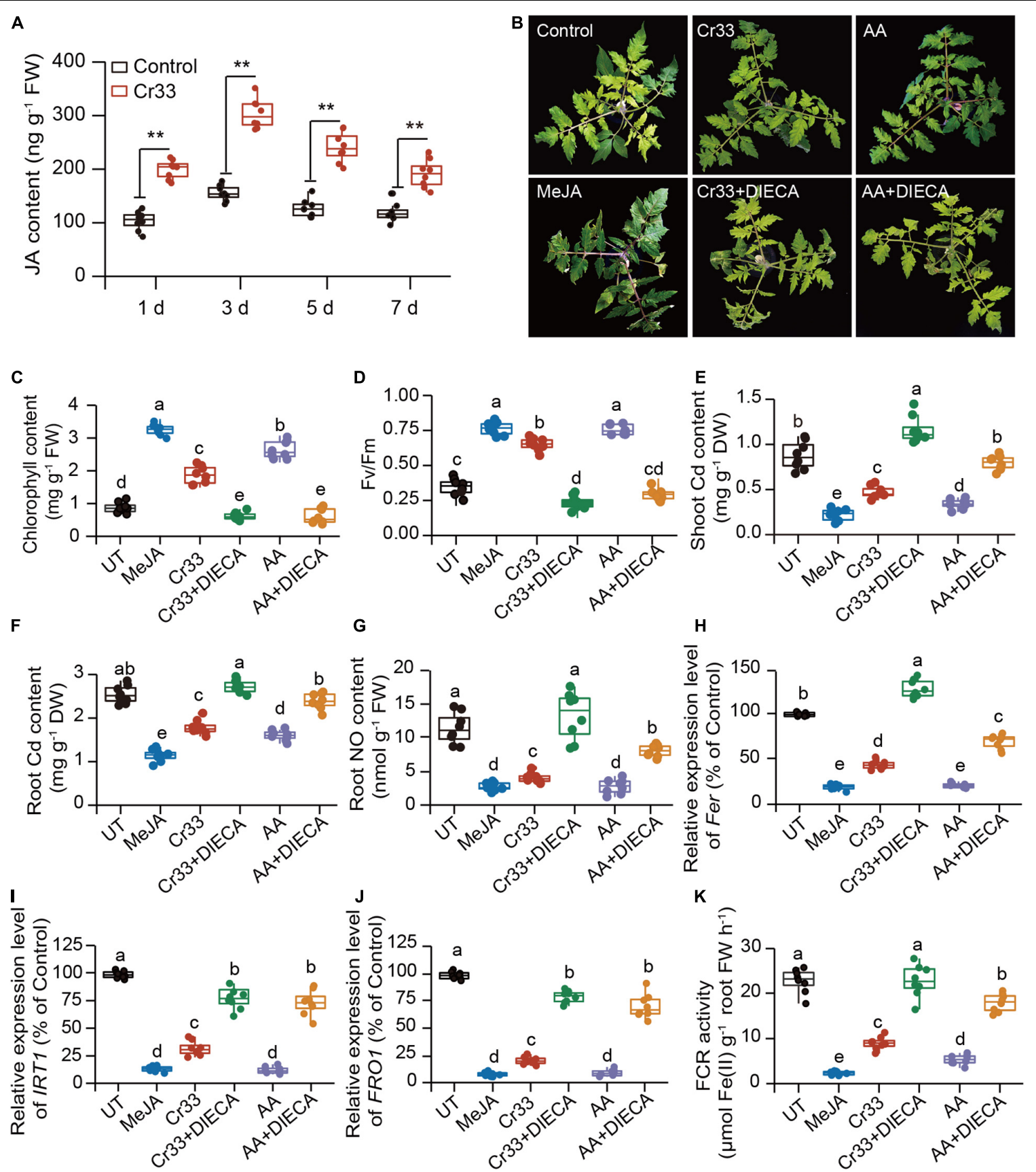

FIGURE 7 | Involvement of JA signals in the L. fusiformis Cr33-induced Cd resistance in tomato plants. (A) Root JA content within 7 d of Cd treatment. Asterisks indicated significant differences between the non-inoculated (control) and inoculated plants $\left(n=8\right.$ biological replicates) using Student's $t$-test $\left({ }^{\star \star} p<0.01\right.$ ). Three-weeks-old tomato plants were exposed to $100 \mu \mathrm{M} \mathrm{Cd}$ with or without cell suspensions of Cr33 at the final density of $5 \times 10^{7} \mathrm{CFU}^{\mathrm{mL}}{ }^{-1}, 0.15 \mathrm{mM}$ acetic acid (AA), 0.05 mM MeJA, Cr33 plus 0.2 mM DIECA (Cr33 + DIECA), and 0.15 mM AA plus 0.2 mM DIECA (AA + DIECA) for 20 d. The untreated (UT) and treated plants were used to analyze shoot phenotypes (B), chlorophyll content (C), Fv/Fm values (D), shoot (E) and root (F) Cd content, root NO content (G). qPCR analyses of the expression of Fer $\mathbf{( H )}$, IRT1 (I) and FRO1 ( $\mathbf{J})$, and root FCR activity (K) after 48 h of treatments. Different letters indicated significant differences among different experimental groups ( $n=8$ biological replicates) using Duncan's multiple range test at $p<0.05$.

The bioavailability of $\mathrm{Cd}$ in the soil is an important factor that affects Cd uptake in plants (Rajkumar et al., 2012; Xu et al., 2018). We found that inoculation with L. fusiformis increased the bioavailability of $\mathrm{Cd}$ in the rhizospheric soils. This raised a question why higher Cd availability did not aggravate the Cd toxicity in the tomato plants. Herein, we also found that the bioavailability of $\mathrm{Fe}$ in the rhizospheric soils was remarkably increased in the L. fusiformis-treated soils compared with the 


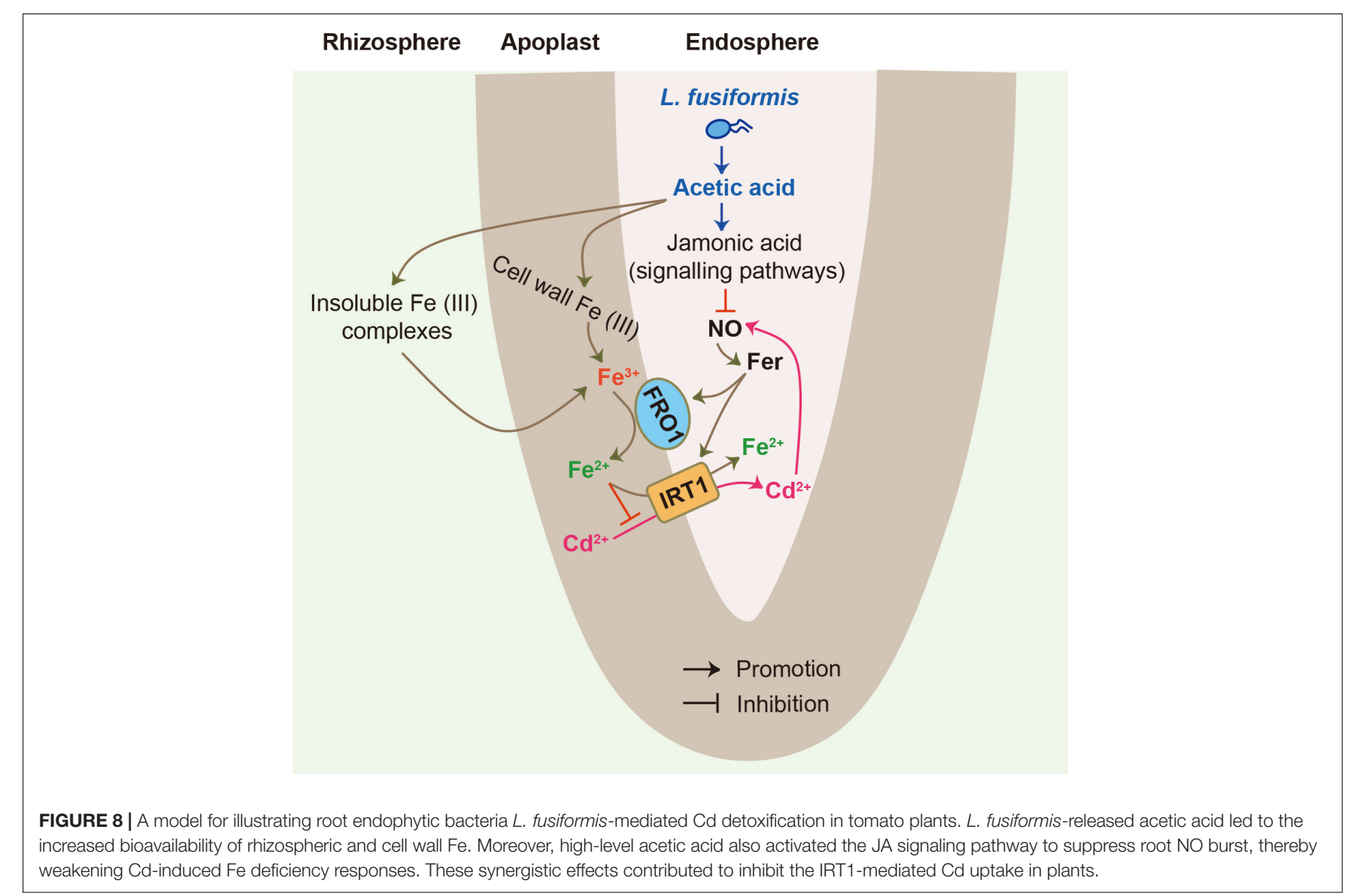

untreated soils. In the hydroponic experiments, root inoculation with $L$. fusiformis also promoted root cell wall Fe remobilization, thereby increasing its availability. Wang et al. (2020) reported that the Cd-tolerant bacteria Burkholderia sp. Y4 prevents Cd uptake in rice by increasing the bioavailability of micronutrients such as $\mathrm{Fe}$ and $\mathrm{Mn}$ in the rhizosphere soils. Cd frequently competes with Fe for the absorption sites and inhibits Fe uptake, thereby provoking Fe deficiency responses (Wu et al., 2012). The increased Fe source can effectively alleviate the Cd toxicity in plants by repressing the root uptake of Cd (Wu et al., 2012; Sebastian and Prasad, 2016; Chen et al., 2017). Hence, the increased bioavailability of $\mathrm{Fe}$ by $L$. fusiformis reinforced the competitiveness of $\mathrm{Fe}$ with $\mathrm{Cd}$ for the absorption sites and further inhibited the entry of Cd into the root cells, which at least partially contributed to less Cd accumulation in the plants.

In this study, soil drenched with L. fusiformis led to higher acetic acid levels in the rhizospheric soil compared with the control plants. Qualitative assays of acetic acid further indicated that L. fusiformis was an acetic acid-producing endophytic bacterium. It is increasingly evidenced that high-level organic acid such as acetic and malic acid can facilitate plant tolerance to adverse conditions such as drought, aluminum (Al) and Cd stress (Hawrylak-Nowak et al., 2015; Pii et al., 2015; Kim et al., 2017). Hence, higher acetic acid levels in the L. fusiformis-inoculated roots may be conducive to alleviating $\mathrm{Cd}$ toxicity in tomato plants. To verify this hypothesis, we investigated the impacts of acetic acid-deficient strains on the Cd-tolerating capacity of the tomato plants. We found that inoculation with acetic acid-deficient strains failed to mitigate Cd stress in the tomato plants. Furthermore, exogenous acetic acid distinctly elevated the capability of plants to tolerate Cd stress. These results strongly supported a pivotal role of acetic acid in detoxifying $\mathrm{Cd}$ in plants. However, the molecular mechanisms of the acetic acid-mediated alleviation of $\mathrm{Cd}$ toxicity in plants have remained elusive thus far.

Besides the increased Fe availability, L. fusiformis may initiate alternative complementary pathways for relieving Cd toxicity in plants. More recently, increased acetic acid level can induce the biosynthesis of JA and thus improve plant drought resistance (Kim et al., 2017). Herein, the biosynthesis of JA in plants was substantially induced by L. fusiformis. Similar results were also observed for the acetic acid-treated tomato plants. We further monitored the responses of L. fusiformis-inoculated plants to Cd stress in the presence of the JA biosynthetic inhibitor DIECA. It was clearly observed that L. fusiformis failed to relieve Cd toxicity in the DIECA-treated plants, indicating that the enhanced JA synthesis was responsible for the L. fusiformisinduced $\mathrm{Cd}$ resistance in the plants. In fact, $\mathrm{Cd}$ toxicity is mainly as a consequence of the dysfunction of essential element absorption, especially $\mathrm{Fe}$, since $\mathrm{Cd}$ stress induces leaf chlorosis and the molecular responses resembling those triggered by 
Fe deficiency (Clemens, 2006; Wu et al., 2012; Sebastian and Prasad, 2016; Chen et al., 2017). Fe deficiency often stimulates the production of $\mathrm{NO}$, which is essential for enhancing the transcription of Fe uptake-related genes in plants (Graziano and Lamattina, 2007; Zhou et al., 2019b). Cd stress can induce root NO burst in many plant species such as Arabidopsis and wheat (Bartha et al., 2005; Valentovicova et al., 2010; ArasimowiczJelonek et al., 2011; Lei et al., 2020). Nevertheless, overproduction of $\mathrm{NO}$ in the roots intensifies the Cd toxicity in Arabidopsis plants, which is positively related to the enhancement of $\mathrm{Cd}$ uptake (Besson-Bard et al., 2009). Herein, Cd stress considerably triggered NO burst in the tomato roots within several hours, whereas the L. fusiformis-inoculated plants exhibited less root $\mathrm{NO}$ accumulation. In accordance with this, the expression of Fe uptake-related genes was greatly weakened in the L. fusiformisinoculated roots under Cd stress compared with the control plants. Reductions in IRT1 mRNA transcripts have been reported to interdict root uptake of Cd (Zhu et al., 2012). Thus, the suppression of root NO burst by L. fusiformis effectively minimized the activation of IRT1 expression in plants, thereby reducing $\mathrm{Cd}$ accumulation. However, treatment with DIECA almost abolished the L. fusiformis-mediated inhibition of root NO burst in the Cd-treated plants. It has recently been indicated that exogenous JA reduces root NO levels in the Cd-stressed plants, thereby down-regulating the expression of AtIRT1 (Lei et al., 2020). Consistently, the Cd-stressed tomato plants exhibited lower root NO levels and IRT1 expression after JA treatment. Hence, our results suggested that the JA-mediated repression of NO signals was responsible for the L. fusiformis-inhibited Cd uptake in the plants.

\section{CONCLUSION}

In summary, although endophytic bacteria have previously been reported to improve plant health and survival under adverse conditions, the mechanisms underpinning these beneficial services have been sparsely explored. Herein, an illustrated model was provided for endophyte-mediated Cd detoxification in tomato plants (Figure 8), in which the L. fusiformis-derived acetic acid resulted in increases in Fe bioavailability and acetic acid levels in the roots. Moreover, high-level acetic acid provoked the JA signaling pathway to inhibit root NO burst, thus attenuating the root $\mathrm{Fe}$ deficiency responses imposed by $\mathrm{Cd}$ stress. Consequently, these synergistic effects contributed to hampering the entry of $\mathrm{Cd}$ into root cells and thus mitigating $\mathrm{Cd}$ toxicity in plants: (1) the increased Fe bioavailability enhanced the competitiveness of Fe with $\mathrm{Cd}$ for metal transporters such

\section{REFERENCES}

Arasimowicz-Jelonek, M., Floryszak-Wieczorek, J., and Gwóźdź, E. A. (2011). The message of nitric oxide in cadmium challenged plants. Plant Sci. 181, 612-620. doi: 10.1016/j.plantsci.2011.03.019

Bal, H. B., Das, S., Dangar, T. K., and Adhya, T. K. (2013). ACC deaminase and IAA producing growth promoting bacteria from the rhizosphere soil of tropical rice plants. J. Basic Microbiol. 53, 972-984. doi: 10.1002/jobm.201200445 as IRT1; and (2) the repression of NO signals down-regulated Fe uptake-related genes under $\mathrm{Cd}$ stress conditions, thereby inhibiting the IRT1-mediated Cd uptake. Therefore, our findings provide novel insights into the mechanisms of endophytemediated Cd detoxification in plants.

\section{DATA AVAILABILITY STATEMENT}

The original contributions presented in the study are publicly available. This data can be found here: NCBI SRA database (No. PRJNA695320).

\section{AUTHOR CONTRIBUTIONS}

CZ and SW: conceptualization and supervision. LZ and JG: investigation and formal analysis, experiments, and analysis of results. CZ: funding acquisition. LZ, JG, and YS: writing original draft. CZ, YS, JG, and CZ: review and editing. All authors contributed to the article and approved the submitted version.

\section{FUNDING}

This work was supported by the China Postdoctoral Science Foundation (No. 2017M620214), the Outstanding Talent Cultivation Program in Colleges and Universities of Education Department of Anhui province (No. gxypZD2020038), the Key Research and Development Projects of Anhui Province (No. 202004a06020003), the Open Project of Key Laboratory of Bio-organic Fertilizer Creation of Ministry of Agriculture and Rural Affairs (No. BOFA202012), and the Natural Science Foundation of Education Department of Anhui province (No. KJ2018ZD051).

\section{ACKNOWLEDGMENTS}

We thank LetPub (www.letpub.com) for its linguistic assistance during the preparation of this manuscript.

\section{SUPPLEMENTARY MATERIAL}

The Supplementary Material for this article can be found online at: https://www.frontiersin.org/articles/10.3389/fpls.2021. 670216/full\#supplementary-material

Balestri, M., Ceccarini, A., Forino, L. M., Zelko, I., Martinka, M., Lux, A., et al. (2014). Cadmium uptake, localization and stress-induced morphogenic response in the fern Pteris vittata. Planta 239, 1055-1064.

Bartha, B., Kolbert, Z., and Erdei, L. (2005). Nitric oxide production induced by heavy metals in Brassica juncea L. Czern. and Pisum sativum L. Acta Biol. Szeged. $49,9-12$.

Besson-Bard, A., Gravot, A., Richaud, P., Auroy, P., Duc, C., Gaymard, F., et al. (2009). Nitric oxide contributes to cadmium toxicity in Arabidopsis 
by promoting cadmium accumulation in roots and by up-regulating genes related to iron uptake. Plant Physiol. 2009, 1302-1315. doi: 10.1104/pp.108.1 33348

Buet, A., Galatro, A., Ramos-Artuso, F., and Simontacchi, M. (2019). Nitric oxide and plant mineral nutrition: current knowledge. J. Exp. Bot. 70, 4461-4476. doi: $10.1093 /$ jxb/erz129

Cassin, G., Mari, S., Curie, C., Briat, J. F., and Czernic, P. (2009). Increased sensitivity to iron deficiency in Arabidopsis thaliana over accumulating nicotianamine. J. Exp. Bot. 60, 1249-1259. doi: 10.1093/jxb/erp007

Chen, X., Yang, Y., Liu, D., Zhang, C., and Ge, Y. (2015). Do soil Fe transformation and secretion of low-molecular-weight organic acids affect the availability of Cd to rice? Environ. Sci. Pollut. R. 22, 19497-19506. doi: 10.1007/s11356-0155134-y

Chen, Z., Tang, Y. T., Yao, A. J., Cao, J., Wu, Z. H., Peng, Z. R., et al. (2017). Mitigation of $\mathrm{Cd}$ accumulation in paddy rice (Oryza sativa L.) by $\mathrm{Fe}$ fertilization. Environ. Pollut. 23, 549-559. doi: 10.1016/j.envpol.2017.08.055

Chen, Z. Z., Xue, C. H., Zhu, S., Zhou, F. F., Ling, X. B., Liu, G. P., et al. (2005). Go pipe: streamlined gene ontology annotation for batch anonymous sequences with statistics. Prog. Biochem. Biophys. 32, 187-191.

Cheng, Y. T., Zhang, L., and He, S. Y. (2019). Plant-microbe interactions facing environmental challenge. Cell Host Microb. 26, 183-192. doi: 10.1016/j.chom. 2019.07.009

Clemens, S. (2006). Toxic metal accumulation, responses to exposure and mechanisms of tolerance in plants. Biochimie 88, 1707-1719. doi: 10.1016/j. biochi.2006.07.003

Gill, S. S., and Tuteja, N. (2011). Cadmium stress tolerance in crop plants: probing the role of sulfur. Plant Signal. Behav. 6, 215-222. doi: 10.4161/psb.6.2.14880

Gouda, S., Kerry, R. G., Das, G., Paramithiotis, S., Shin, H. S., and Patra, J. K. (2018). Revitalization of plant growth promoting rhizobacteria for sustainable development in agriculture. Microbiol. Res. 206, 131-140. doi: 10.1016/j.micres. 2017.08.016

Graziano, M., and Lamattina, L. (2007). Nitric oxide accumulation is required for molecular and physiological responses to iron deficiency in tomato roots. Plant J. 52, 949-960. doi: 10.1111/j.1365-313x.2007.03283.x

Grusak, M. A. (1995). Whole-root iron(III)-reductase activity throughout the life cycle of iron-grown Pisum sativum L. (Fabaceae): relevance to the iron nutrition of developing seeds. Planta 197, 111-117.

Han, X., Zhang, Y., Yu, M., Zhang, J., Xu, D., Lu, Z., et al. (2020). Transporters and ascorbate-glutathione metabolism for differential cadmium accumulation and tolerance in two contrasting willow genotypes. Tree Physiol. 40, 1126-1142. doi: 10.1093/treephys/tpaa029

Hardoim, P. R., van Overbeek, L. S., Berg, G., Pirttilä, A. M., Compant, S., Campisano, A., et al. (2015). The hidden world within plants: ecological and evolutionary considerations for defining functioning of microbial endophytes. Microbiol. Mol. Biol. Rev. 79, 293-320. doi: 10.1128/mmbr.00050- 14

Hawrylak-Nowak, B., Dresler, S., and Matraszek, R. (2015). Exogenous malic and acetic acids reduce cadmium phytotoxicity and enhance cadmium accumulation in roots of sunflower plants. Plant Physiol. Biochem. 94, 225-234. doi: 10.1016/j.plaphy.2015.06.012

Jin, C. W., You, G. Y., He, Y. F., Tang, C., Wu, P., and Zheng, S. J. (2007). Iron deficiency-induced secretion of phenolics facilitates the reutilization of root apoplastic iron in red clover. Plant Physiol. 144, 278-285. doi: 10.1104/pp.107. 095794

Kim, J. M., To, T. K., Matsui, A., Tanoi, K., Kobayashi, N. I., Matsuda, F., et al. (2017). Acetate-mediated novel survival strategy against drought in plants. Nat. Plants 3:17097.

Lei, G. J., Sun, L., Sun, Y., Zhu, X. F., Li, G. X., and Zheng, S. J. (2020). Jasmonic acid alleviates cadmium toxicity in Arabidopsis via suppression of cadmium uptake and translocation. J. Integr. Plant Biol. 62, 218-227. doi: 10.1111/jipb.1 2801

Lei, G. J., Yokosho, K., Yamaji, N., and Ma, J. F. (2017). Two MATE transporters with different subcellular localization are involved in $\mathrm{Al}$ tolerance in buckwheat. Plant Cell Physiol. 58, 2179-2189. doi: 10.1093/pcp/p cx152

Lei, G. J., Zhu, X. F., Wang, Z. W., Dong, F., Dong, N. Y., and Zheng, S. J. (2014). Abscisic acid alleviates iron deficiency by promoting root iron reutilization and transport from root to shoot in Arabidopsis. Plant Cell Environ. 37, 852-863. doi: $10.1111 /$ pce. 12203
Lešková, A., Giehl, R. F. H., Hartmann, A., Fargašová, A., and von Wirén, N. (2017). Heavy metals induce iron deficiency responses at different hierarchic and regulatory levels. Plant Physiol. 174, 1648-1668. doi: 10.1104/pp.16.01916

Liu, Y., Zhu, A., Tan, H., Cao, L., and Zhang, R. (2019). Engineering banana endosphere microbiome to improve Fusarium wilt resistance in banana. Microbiome 7:74.

Lugtenberg, B., and Kamilova, F. (2009). Plant-growth-promoting rhizobacteria. Annu. Rev. Microbiol. 63, 541-556.

Mao, X. Z., Cai, T., Olyarchuk, J. G., and Wei, L. P. (2005). Automated genome annotation and pathway identification using the KEGG Orthology (KO) as a controlled vocabulary. Bioinformatics 2, 3787-3793. doi: 10.1093/ bioinformatics/bti430

Montiel-Rozas, M. M., Madejón, E., and Madejón, P. (2016). Effect of heavy metals and organic matter on root exudates (low molecular weight organic acids) of herbaceous species: An assessment in sand and soil conditions under different levels of contamination. Environ. Pollut. 216, 273-281. doi: 10.1016/j.envpol. 2016.05.080

Oleńska, E., Małek, W., Wójcik, M., Swiecicka, I., Thijs, S., and Vangronsveld, J. (2020). Beneficial features of plant growth-promoting rhizobacteria for improving plant growth and health in challenging conditions: A methodical review. Sci. Total Environ. 743:140682. doi: 10.1016/j.scitotenv.2020.140682

Pan, W., Lu, Q., Xu, Q. R., Zhang, R. R., Li, H. Y., Yang, Y. H., et al. (2019). Abscisic acid-generating bacteria can reduce $\mathrm{Cd}$ concentration in pakchoi grown in Cd-contaminated soil. Ecotoxicol. Environ. Saf. 177, 100-107.

Pii, Y., Penn, A., Terzano, R., Crecchio, C., Mimmo, T., and Cesco, S. (2015). Plantmicroorganism-soil interactions influence the $\mathrm{Fe}$ availability in the rhizosphere of cucumber plants. Plant Physiol. Biochem. 87, 45-52. doi: 10.1016/j.plaphy. 2014.12.014

Porra, R. J. (2002). The chequered history of the development and use of simultaneous equations for the accurate determination of chlorophylls a and b. Photosynth. Res. 73, 149-156. doi: 10.4324/9781351187596-10

Pulford, I. D., and Watson, C. (2003). Phytoremediation of heavy metal contaminated land by trees-a review. Environ. Int. 29, 529-540. doi: 10.1016/ s0160-4120(02)00152-6

Rajkumar, M., Sandhya, S., Prasad, M. N., and Freitas, H. (2012). Perspectives of plant-associated microbes in heavy metal phytoremediation. Biotechnol. Adv. 30, 1562-1574. doi: 10.1016/j.biotechadv.2012.04.011

Ravanbakhsh, M., Kowalchuk, G. A., and Jousset, A. (2019). Optimization of plant hormonal balance by microorganisms prevents plant heavy metal accumulation. J. Hazard. Mater. 379:120787. doi: 10.1016/j.jhazmat.2019. 120787

Sarwar, N., Imran, M., Shaheen, M. R., Ishaque, W., Kamran, M. A., and Matloob, A. (2017). Phytoremediation strategies for soils contaminated with heavy metals: modifications and future perspectives. Chemosphere 171, 710-721. doi: 10.1016/j.chemosphere.2016.12.116

Schützendübel, A., and Polle, A. (2002). Plant responses to abiotic stresses: heavy metal-induced oxidative stress and protection by mycorrhization. J. Exp. Bot. 53, 1351-1365. doi: 10.1093/jexbot/53.372.1351

Sebastian, A., and Prasad, M. N. (2016). Iron plaque decreases cadmium accumulation in Oryza sativa L. and serves as a source of iron. Plant Biol. 18, 1008-1015. doi: 10.1111/plb.12484

Sharifi, R., and Ryu, C. M. (2018). Revisiting bacterial volatile-mediated plant growth promotion: lessons from the past and objectives for the future. Ann. Bot. 122, 349-358. doi: 10.1093/aob/mcy108

Tanwir, K., Javed, M. T., Abbas, S., Shahid, M., Akram, M. S., Chaudhary, H. J., et al. (2021). Serratia sp. CP-13 alleviates Cd toxicity by morpho-physio-biochemical improvements, antioxidative potential and diminished Cd uptake in Zea mays L. cultivars differing in Cd tolerance. Ecotoxicol. Environ. Saf. 208:111584. doi: 10.1016/j.ecoenv.2020.111584

Valentovicova, K., Haluskova, L., Huttova, J., Mistrık, I., and Tamas, L. (2010). Effect of cadmium on diaphorase activity and nitric oxide production in barley root tips. J. Plant Physiol. 167, 10-14. doi: 10.1016/j.jplph.2009.06.018

Wang, C. G., Huang, Y. C., Yang, X. R., Xue, W. J., Zhang, X., Zhang, Y. H., et al. (2020). Burkholderia sp. Y4 inhibits cadmium accumulation in rice by increasing essential nutrient uptake and preferentially absorbing cadmium. Chemosphere 252:126603. doi: 10.1016/j.chemosphere.2020.126603

Wang, H., Liang, X., Huang, J., Zhang, D., Lu, H., Liu, Z., et al. (2010). Involvement of ethylene and hydrogen peroxide in induction of alternative respiratory 
pathway in salt-treated Arabidopsis calluses. Plant Cell Physiol. 51, 1754-1765. doi: $10.1093 / \mathrm{pcp} / \mathrm{pcq} 134$

Wu, H., Chen, C., Du, J., Liu, H., Cui, Y., Zhang, Y., et al. (2012). Co-overexpression FIT with AtbHLH38 or AtbHLH39 in Arabidopsis enhanced cadmium tolerance via increased cadmium sequestration in roots and improved iron homeostasis of shoots. Plant Physiol. 158, 790-800. doi: 10.1104/pp.111.190983

Wu, S., Zhuang, G., Bai, Z., Cen, Y., Xu, S., Sun, H., et al. (2018). Mitigation of nitrous oxide emissions from acidic soils by Bacillus amyloliquefaciens, a plant growth-promoting bacterium. Glob. Chang Biol. 24, 2352-2365.

Xu, Q., Pan, W., Zhang, R., Lu, Q., Xue, W., Wu, C., et al. (2018). Inoculation with Bacillus subtilis and Azospirillum brasilense produces abscisic acid that reduces Irt1-mediated cadmium uptake of roots. J. Agric. Food Chem. 66, 5229-5236. doi: $10.1021 /$ acs.jafc.8b00598

Xu, Z., Wang, M., Xu, D., and Xia, Z. (2020). The Arabidopsis APR2 positively regulates cadmium tolerance through glutathione-dependent pathway. Ecotoxicol. Environ. Saf. 187:109819. doi: 10.1016/j.ecoenv.2019.109819

Zhou, C., Ge, N. G., Guo, J. S., Zhu, L., Ma, Z. Y., Cheng, S. Y., et al. (2019a). Enterobacter asburiae reduces cadmium toxicity in maize plants by repressing iron uptake-associated pathways. J. Agric. Food Chem. 67, 10126-10136. doi: 10.1021/acs.jafc.9b03293
Zhou, C., Zhu, L., Guo, J., Xiao, X., Ma, Z., and Wang, J. (2019b). Bacillus subtilis STU6 ameliorates iron deficiency in tomato by enhancement of polyaminemediated iron remobilization. J. Agric. Food Chem. 67, 320-330. doi: 10.1021/ acs.jafc. 8 b05851

Zhu, X. F., Jiang, T., Wang, Z. W., Lei, G. J., Shi, Y. Z., Li, G. X., et al. (2012). Gibberellic acid alleviates cadmium toxicity by reducing nitric oxide accumulation and expression of IRT1 in Arabidopsis thaliana. J. Hazard. Mater. 23, 302-307. doi: 10.1016/j.jhazmat.2012 .08 .077

Conflict of Interest: The authors declare that the research was conducted in the absence of any commercial or financial relationships that could be construed as a potential conflict of interest.

Copyright $\odot 2021 \mathrm{Zhu}$, Guo, Sun, Wang and Zhou. This is an open-access article distributed under the terms of the Creative Commons Attribution License (CC BY). The use, distribution or reproduction in other forums is permitted, provided the original author(s) and the copyright owner(s) are credited and that the original publication in this journal is cited, in accordance with accepted academic practice. No use, distribution or reproduction is permitted which does not comply with these terms. 